\title{
Odd linking and bifurcation in gaps: the weakly indefinite case
}

\author{
Hans-Jörg Ruppen \\ École Polytechnique Fédérale de Lausanne, \\ Cours de Mathématiques Spéciale, Station 4, \\ 1015 Lausanne, Switzerland (hans-joerg.ruppen@epfl.ch)
}

(MS received 10 October 2011; accepted 8 August 2012)

In this paper, we consider nonlinear Schrödinger equations of the following type: $-\Delta u(x)+V(x) u(x)-q(x)|u(x)|^{\sigma} u(x)=\lambda u(x), \quad x \in \mathbb{R}^{N}, u \in H^{1}\left(\mathbb{R}^{N}\right) \backslash\{0\}$,

where $N \geqslant 2$ and $\sigma>0$. We concentrate on situations where the potential function $V$ appearing in the linear part of the equation is of Coulomb type; by this we mean potentials where the spectrum of the linear operator $-\Delta+V$ consists of an increasing sequence of eigenvalues $\lambda_{1}, \lambda_{2}, \ldots$ followed by an interval belonging to the essential spectrum.

We study, for $\lambda$ kept fixed inside a spectral gap or below $\lambda_{1}$, the existence of multiple solution pairs, as well as the bifurcation behaviour of these solutions when $\lambda$ approaches a point of the spectrum from the left-hand side. Our method proceeds by an analysis of critical points of the corresponding energy functional. To this end, we derive a new variational characterization of critical levels

$c_{0}(\lambda) \leqslant c_{1}(\lambda) \leqslant c_{2}(\lambda) \leqslant \cdots$ corresponding to an infinite set of critical points.

We derive such a multiplicity result even if some of the critical values $c_{n}(\lambda)$ coincide; this seems to be a major advantage of our approach. Moreover, the characterization of these values $c_{n}(\lambda)$ is suitable for an analysis of the bifurcation behaviour of the corresponding generalized solutions.

The approach presented here is generic; for instance, it can be applied when $V$ and $q$ are periodic functions. Such generalizations are briefly described in this paper and will be the object of a forthcoming article.

\section{Introduction}

In this paper, we investigate nonlinear Schrödinger equations of the following type:

$$
\left.\begin{array}{l}
-\Delta u(x)+V(x) u(x)-q(x)|u(x)|^{\sigma} u(x)=\lambda u(x), \quad x \in \mathbb{R}^{N}, \\
u \in H^{1}\left(\mathbb{R}^{N}\right) \backslash\{0\},
\end{array}\right\}
$$

where $N \geqslant 2$ and $\sigma>0$. The nonlinear perturbation $q(x)|u(x)|^{\sigma} u(x)$ is governed by a positive function $q$. Therefore, we call it a simple nonlinearity, as opposed to other nonlinearities like $q(x)|u(x)|^{\sigma} u(x)-r(x)|u(x)|^{\tau} u(x)$, where both $q$ and $r$ are positive functions and $0<\sigma<\tau$ (see, for example, [10,11]).

We include in our analysis the important 'hydrogen-like' situations. Thus, we consider potentials $V$ for which the spectrum of the linear operator $-\Delta+V$ consists of an increasing set of eigenvalues $\lambda_{1}, \lambda_{2}, \ldots$ followed by an interval belonging to the essential spectrum. Our analysis deals not only with situations where this set

(C) 2013 The Royal Society of Edinburgh 
of eigenvalues is finite, but also with the important case of a countable number of eigenvalues. A typical example of such a potential is the Coulomb potential $V(x)=-2 /|x|$. We will treat the case where $\lambda$ lies below $\lambda_{1}$, as well as the more delicate one where $\lambda$ lies inside a spectral gap. As well as the existence of multiple solutions for a fixed value of $\lambda$, we will study the bifurcation behaviour of such solutions when $\lambda$ varies.

The paper has the following structure.

In $\S 2$, we set up the basic hypotheses under which we analyse (1.1). Moreover, we explain what we mean by a solution; we restrict our attention to the existence of weak solutions characterized as critical points of some energy functional $I_{\lambda}$. In doing so, we are led in a natural way to an extension $L_{1}+V$ of the operator $-\Delta+V$. Section 3 deals with the invariance of the point spectrum when replacing $-\Delta+V$ by its extension $L_{1}+V$.

In $\S 4$, we show that the energy functional $I_{\lambda}$ satisfies a compactness assumption known as the Palais-Smale condition. Note that this assumption holds only on the resolvent set. Therefore, we must exclude questions about the existence of solutions when $\lambda$ belongs to the spectrum of $-\Delta+V$.

In $\S 5$, we present the main abstract result; we study critical points of a functional $I_{\lambda}: X \rightarrow \mathbb{R}$ that is defined on an abstract Hilbert space $X$ and that depends on a parameter $\lambda$. This functional consists of two terms: a quadratic one that predominates in a neighbourhood of the origin, and a negative, superquadratic one whose importance increases with the distance from the origin. We deal with a setting where the space $X$ is split into an orthogonal sum $Y \oplus Z$; the quadratic term of $I_{\lambda}$ is negative definite on $Y$ and positive definite on $Z$. Thereby, the subspace $Y$ is finite-dimensional; we call such situations 'weakly indefinite' in contrast to the 'strongly indefinite' ones, where $\operatorname{dim} Y=\infty$ (see $[12,13])$.

It would be possible to use here, at least for existence results, the classical results by Ambrosetti and Rabinowitz [1]. We choose a different approach and give a new variational characterization of critical levels $c_{0}(\lambda) \leqslant c_{1}(\lambda) \leqslant c_{2}(\lambda) \leqslant \cdots$. As Ambrosetti and Rabinowitz did, we give a multiplicity result when some of the levels $c_{j}(\lambda)(j \in \mathbb{N})$ coincide. Our approach has two major advantages. Firstly, we can easily analyse the behaviour of critical points when the parameter $\lambda$ varies. In this way we get multiple bifurcation results. Secondly, and this seems to be the more important point, we can extend our ideas to the 'strongly indefinite' cases and we will present such situations in a forthcoming paper [12]. This unifying aspect seems to be new and opens the door to the analysis of multiple bifurcation independently of the dimension of the space $Y$.

Section 6 applies the abstract setting to the Schrödinger equation (1.1) when $\lambda$ is below the first eigenvalue. In $\S 7$, we consider the same problem when the eigenvalue $\lambda$ is in a spectral gap. In both cases, we prove the existence of an infinite number of solutions and we derive a (multiple) bifurcation result in each spectral gap.

\section{Basic assumptions}

Our study will include 'hydrogen-like' configurations; by this we mean a setting where a nucleus of mass $m_{1}$ and of charge $Z e$ is surrounded by an electron of mass $m_{2}$ and of charge $-e$. Following Jost [7], the corresponding governing equation takes 
the form

$$
-\Delta u(x)-\frac{2}{|x|} u(x)=\varepsilon u(x), \quad x \in \mathbb{R}^{3},
$$

involving the Coulomb potential $V(x)=-2 /|x|$. It is well known that the eigenvalues of the operator $-\Delta+V$ are $\lambda_{n}=-1 / n^{2}(n=1,2,3, \ldots)$. This fact, together with $\sigma_{e}(-\Delta+V)=\left[0,+\infty\left[\right.\right.$ motivates the following set of assumptions $\mathrm{V}^{-}, \mathrm{V}$ and $\mathrm{L}$.

We begin with a basic assumption involving only the potential function $V$.

Assumption $\mathrm{V}^{-}$. When considered as a mapping $H^{1}\left(\mathbb{R}^{N}\right) \rightarrow L^{2}\left(\mathbb{R}^{N}\right)$, the operator $V: u(x) \mapsto V(x) u(x)$ is continuous. Moreover, $\lim _{|x| \rightarrow+\infty} V(x)=0^{-}$uniformly, i.e. given any $b<0$, there exists an $r_{b}>0$ such that $b / 2 \leqslant V(x) \leqslant 0$ for $|x| \geqslant r_{b}$.

We will need an additional assumption, especially when discussing the PalaisSmale condition.

Assumption $\mathrm{V}$. The potential $V$ satisfies assumption $\mathrm{V}^{-}$. Moreover, when considered as a mapping $H^{2}\left(\mathbb{R}^{N}\right) \rightarrow L^{2}\left(\mathbb{R}^{N}\right)$, the operator $V: u(x) \mapsto V(x) u(x)$ is completely continuous in the sense that $V u_{m} \rightarrow V u$ in $L^{2}\left(\mathbb{R}^{N}\right)$ as $m \rightarrow \infty$ whenever $u_{m} \rightarrow u$ in $H^{2}\left(\mathbb{R}^{N}\right)$ as $m \rightarrow \infty$.

Proposition 2.1. The Coulomb potential $V(x)=-K /|x|$, where $K$ is a positive constant, satisfies assumption $V$.

For a proof, we refer the reader to Dautray and Lions [3, pp. 385-431].

We now proceed with the assumptions on $V$ as part of the operator

$$
-\Delta+V: \boldsymbol{D}(-\Delta+V) \subset L^{2}\left(\mathbb{R}^{N}\right) \rightarrow L^{2}\left(\mathbb{R}^{N}\right),
$$

with $\boldsymbol{D}(-\Delta+V)=\boldsymbol{D}(-\Delta)=H^{2}\left(\mathbb{R}^{N}\right)$. Under assumption $\mathrm{V}$, we have by Weyl's Theorem that

$$
\sigma_{e}(-\Delta+V)=\sigma_{e}(-\Delta)=[0,+\infty[.
$$

Note that $\sigma(-\Delta)=\sigma_{e}(-\Delta)$, but this must no longer be true for the operator $-\Delta+V$. The interested reader can find more details in Dautray and Lions [3, p. 383]. In this paper, we restrict ourselves to situations that satisfy the following condition.

Assumption L. The potential $V$ satisfies assumption $\mathrm{V}$ with $N>1$. Moreover, the operator $-\Delta+V$ has a discrete spectrum (consisting of isolated eigenvalues of finite multiplicity) of the form

$$
\sigma_{d}(-\Delta+V)=\left\{\lambda_{n} \mid n \in L\right\}
$$

where

- $\lambda_{1}<\lambda_{2}<\cdots<0, \lambda_{1}$ being simple,

- $L=\mathbb{N}$ or $L=\{1,2, \ldots, \ell\}$ for some $\ell \in \mathbb{N}$,

- $\lim _{n \rightarrow \infty} \lambda_{n}=0^{-}$if $L=\mathbb{N}$.

Proposition 2.2. The Coulomb potential $V(x)=-2 /|x|$ satisfies assumption $L$ with $L=\mathbb{N}$. 
We now concentrate on the nonlinear perturbation $q|u|^{\sigma} u$. We work under the following assumption.

Assumption Q. The non-negative function $q \in L^{\infty}\left(\mathbb{R}^{N}\right)$ satisfies $\lim _{|x| \rightarrow \infty} q(x)=$ 0 . The constant $\sigma$ is strictly positive. Moreover, $\sigma<4 /(N-2)$ if $N>2$.

Under this assumption, we may consider the functional

$$
\Phi(u):=\frac{1}{2+\sigma} \int_{\mathbb{R}^{N}} q(x)|u(x)|^{2+\sigma} \mathrm{d} x \quad \forall u \in H^{1}\left(\mathbb{R}^{N}\right) .
$$

Before proceeding, let us fix some notation. We denote by $(\cdot, \cdot)_{H^{1}\left(\mathbb{R}^{N}\right)}$ the usual scalar product in $H^{1}\left(\mathbb{R}^{N}\right)$; the corresponding norm will be $\|\cdot\|_{H^{1}\left(\mathbb{R}^{N}\right)}$. Moreover, given a functional $f$ of class $C^{1}$, we define the gradient $\nabla f$ by

$$
f^{\prime}(u) v=(\nabla f(u), v)_{H^{1}\left(\mathbb{R}^{N}\right)}
$$

for all $u, v \in H^{1}\left(\mathbb{R}^{N}\right)$.

The following result can be found in Stuart $[17,18]$.

Proposition 2.3. If assumption $Q$ holds, we have the following.

(1) $\Phi \in C^{1}\left(H^{1}\left(\mathbb{R}^{N}\right) ; \mathbb{R}\right), \Phi^{\prime}(u)=q|u|^{\sigma} u$, where the equality holds in $H^{-1}\left(\mathbb{R}^{N}\right)$.

(2) $\Phi$ is completely continuous in the sense that $\Phi\left(u_{m}\right) \rightarrow \Phi(u)$ in $\mathbb{R}$ as $m \rightarrow \infty$ whenever $u_{m} \rightarrow u$ in $H^{1}\left(\mathbb{R}^{N}\right)$ as $m \rightarrow \infty$.

(3) $\Phi^{\prime}$ is compact in the sense that $\nabla \Phi\left(u_{m}\right) \rightarrow \nabla \Phi(u)$ in $H^{1}\left(\mathbb{R}^{N}\right)$ as $m \rightarrow \infty$ whenever $u_{m} \rightarrow u$ in $H^{1}\left(\mathbb{R}^{N}\right)$ as $m \rightarrow \infty$.

We now turn our attention to the nonlinear Schrödinger equation. Supposing that assumptions L and Q hold, for all $u \in H^{1}\left(\mathbb{R}^{N}\right)$, we set

$$
B_{\lambda}(u):=\int_{\mathbb{R}^{N}}\left(|\nabla u|^{2}+V u^{2}-\lambda u^{2}\right) \mathrm{d} x \quad \text { and } \quad I_{\lambda}(u):=\frac{1}{2} B_{\lambda}(u)-\Phi(u) .
$$

Problem (1.1) can be formulated in a weak form as follows.

Problem P. For $\lambda \in]-\infty, 0\left[\backslash \sigma(-\Delta+V)\right.$ kept fixed, find a function $u \in H^{1}\left(\mathbb{R}^{N}\right) \backslash$ $\{0\}$, with $I_{\lambda}^{\prime}(u)=0$ in $H^{-1}\left(\mathbb{R}^{N}\right)$.

Note that any solution $u$ of problem $\mathrm{P}$ must verify $B_{\lambda}(u)>0$. This is so, since $I_{\lambda}^{\prime}(u) u=B_{\lambda}(u)-(2+\sigma) \Phi(u)=0$ implies that

$$
I_{\lambda}(u)=\left(\frac{1}{2}-\frac{1}{2+\sigma}\right) B_{\lambda}(u)=\left(\frac{2+\sigma}{2}-1\right) \Phi(u)>0 .
$$

\section{Analysis of the linear term}

We begin the study of problem $\mathrm{P}$ with the analysis of the linear term that generates the quadratic part $B_{\lambda}$ of the energy functional $I_{\lambda}$. We consider the positive, selfadjoint operator

$$
-\Delta: H^{2}\left(\mathbb{R}^{N}\right) \subset L^{2}\left(\mathbb{R}^{N}\right) \rightarrow L^{2}\left(\mathbb{R}^{N}\right)
$$


and its positive, self-adjoint square-root

$$
T:=(-\Delta)^{1 / 2}: H^{1}\left(\mathbb{R}^{N}\right) \subset L^{2}\left(\mathbb{R}^{N}\right) \rightarrow L^{2}\left(\mathbb{R}^{N}\right)
$$

(see Kato [8, pp. 281 and 331]). Stuart [15-17] devoted a couple of articles to such operators; we summarize his main results here.

For $u \in H^{2}\left(\mathbb{R}^{N}\right)$ and $v \in H^{1}\left(\mathbb{R}^{N}\right)$, we have that

$$
(T u, T v)_{L^{2}\left(\mathbb{R}^{N}\right)}=\left(T^{2} u, v\right)_{L^{2}\left(\mathbb{R}^{N}\right)}=(-\Delta u, v)_{L^{2}\left(\mathbb{R}^{N}\right)}=(\nabla u, \nabla v)_{L^{2}\left(\mathbb{R}^{N}\right)} .
$$

Using a density argument, we may thus conclude that the bilinear form

$$
a(u, v)=\int_{\mathbb{R}^{N}} \nabla u \cdot \nabla v \mathrm{~d} x
$$

can be written as

$$
a(u, v)=(T u, T v)_{L^{2}\left(\mathbb{R}^{N}\right)} \quad \forall u, v \in H^{1}\left(\mathbb{R}^{N}\right) .
$$

We introduce the bounded, linear operator (of norm $\leqslant 1$ ) defined by

$$
T_{1}: H^{1}\left(\mathbb{R}^{N}\right) \rightarrow L^{2}\left(\mathbb{R}^{N}\right), \quad u \mapsto T u .
$$

Moreover, we set $L_{1}:=T_{1}^{*} T_{1}: H^{1}\left(\mathbb{R}^{N}\right) \rightarrow H^{-1}\left(\mathbb{R}^{N}\right)$. Note that we make use of the identification

$$
H^{1}\left(\mathbb{R}^{N}\right) \subset L^{2}\left(\mathbb{R}^{N}\right)=\left[L^{2}\left(\mathbb{R}^{N}\right)\right]^{*} \subset H^{-1}\left(\mathbb{R}^{N}\right) .
$$

Then, we have the following.

(1) $L_{1}$ is an extension of $-\Delta$ and

$$
u \in H^{2}\left(\mathbb{R}^{N}\right) \Longleftrightarrow L_{1} u \in L^{2}\left(\mathbb{R}^{N}\right) .
$$

(2) $a(u, v)=\left\langle L_{1} u, v\right\rangle_{H^{-1}\left(\mathbb{R}^{N}\right), H^{1}\left(\mathbb{R}^{N}\right)} \quad \forall u, v \in H^{1}\left(\mathbb{R}^{N}\right)$.

Proposition 3.1. For $\lambda<0$, the operator $L_{1}-\lambda \mathbb{I}: H^{1}\left(\mathbb{R}^{N}\right) \rightarrow H^{-1}\left(\mathbb{R}^{N}\right)$ is invertible (with a bounded inverse).

Proof. We divide the proof into three steps. The whole procedure is deeply inspired by Heinz [5].

STEP 1. Let us consider the positive, self-adjoint operator

$$
M:=-\Delta+\mathbb{I}: H^{2}\left(\mathbb{R}^{N}\right) \subset L^{2}\left(\mathbb{R}^{N}\right) \rightarrow L^{2}\left(\mathbb{R}^{N}\right)
$$

and let us set, for $\alpha>0$,

- $M_{\alpha}:=(-\Delta+\mathbb{I})^{\alpha}$ and $M_{-\alpha}:=\left(M_{\alpha}\right)^{-1}$,

- $H^{\alpha}:=\boldsymbol{D}\left(M_{\alpha / 2}\right)$ equipped with the norm $\|u\|_{\alpha}:=\left|M_{\alpha / 2} u\right|_{L^{2}\left(\mathbb{R}^{N}\right)}$.

Denoting by $E(\lambda)$ the decomposition of the identity corresponding to $M$ (see Kato [8, pp. 353-356]), we may then write

$M=\int_{1}^{+\infty} \lambda \mathrm{d} E(\lambda), \quad M_{1 / 2}=\int_{1}^{+\infty} \sqrt{\lambda} \mathrm{d} E(\lambda) \quad$ and $\quad-\Delta=\int_{1}^{+\infty}(\lambda-1) \mathrm{d} E(\lambda)$. 
Hence, we get that

$$
\begin{aligned}
H^{1}\left(\mathbb{R}^{N}\right) & =\left\{u \in L^{2}\left(\mathbb{R}^{N}\right) \mid \int_{1}^{+\infty}(\lambda-1) \mathrm{d}(E(\lambda) u, u)_{L^{2}\left(\mathbb{R}^{N}\right)}<\infty\right\} \\
& =\boldsymbol{D}\left(M_{1 / 2}\right)=H^{1}
\end{aligned}
$$

and, in a similar way, we may derive that $H^{2}=H^{2}\left(\mathbb{R}^{N}\right)$.

Moreover, using a density argument and the relation $\|u\|_{1}^{2}=(M u, u)_{L^{2}\left(\mathbb{R}^{N}\right)}=$ $(-\Delta u+u, u)_{L^{2}\left(\mathbb{R}^{N}\right)}=\|u\|_{H^{1}\left(\mathbb{R}^{N}\right)}^{2}\left(\right.$ for $\left.u \in H^{2}\right)$, we get that $\|u\|_{1}=\|u\|_{H^{1}\left(\mathbb{R}^{N}\right)}$ for all $u \in H^{1}\left(\mathbb{R}^{N}\right)$.

STEP 2. We now look at the operator

$$
N:=T_{1}^{*} T_{1}+\mathbb{I}: H^{1}\left(\mathbb{R}^{N}\right) \rightarrow H^{-1}\left(\mathbb{R}^{N}\right),
$$

and we show that, for all $\lambda<1, N-\lambda \mathbb{I}: H^{1}\left(\mathbb{R}^{N}\right) \rightarrow H^{-1}\left(\mathbb{R}^{N}\right)$ is invertible and has a unique inverse.

Indeed, since $\lambda-1 \notin \sigma(-\Delta)=[0,+\infty[$, the operator

$$
R:=(-\Delta-(\lambda-1) \mathbb{I})^{-1}: L^{2}\left(\mathbb{R}^{N}\right) \rightarrow L^{2}\left(\mathbb{R}^{N}\right)
$$

is well defined and bounded. So

$$
M R=(-\Delta+\mathbb{I})(-\Delta+\mathbb{I}-\lambda \mathbb{I})^{-1}=\mathbb{I}+\lambda R: L^{2}\left(\mathbb{R}^{N}\right) \rightarrow L^{2}\left(\mathbb{R}^{N}\right)
$$

is also well defined and bounded. Note that, for $v \in L^{2}\left(\mathbb{R}^{N}\right), R v \in H^{2}\left(\mathbb{R}^{N}\right) \subset$ $H^{1}\left(\mathbb{R}^{N}\right)$. So

$$
\begin{aligned}
\|R v\|_{H^{1}\left(\mathbb{R}^{N}\right)}^{2} & =\|R v\|_{1}^{2}=\left|M_{1 / 2} R v\right|_{L^{2}\left(\mathbb{R}^{N}\right)}^{2}=\left|M R M_{-1 / 2} v\right|_{L^{2}\left(\mathbb{R}^{N}\right)}^{2} \\
& \leqslant\|M R\|^{2}\left|M_{-1 / 2} v\right|_{L^{2}\left(\mathbb{R}^{N}\right)}
\end{aligned}
$$

But,

$$
\begin{aligned}
\left|M_{-1 / 2} v\right|_{L^{2}\left(\mathbb{R}^{N}\right)}^{2} & =\left(v, M_{-1} v\right)_{L^{2}\left(\mathbb{R}^{N}\right)} \leqslant\|v\|_{H^{-1}\left(\mathbb{R}^{N}\right)}\left\|M_{-1} v\right\|_{1} \\
& =\|v\|_{H^{-1}\left(\mathbb{R}^{N}\right)}\left|M_{-1 / 2} v\right|_{L^{2}\left(\mathbb{R}^{N}\right)} .
\end{aligned}
$$

Hence, we get that

$$
\|R v\|_{H^{1}\left(\mathbb{R}^{N}\right)} \leqslant\|M R\|\|v\|_{H^{-1}\left(\mathbb{R}^{N}\right)} \quad \forall v \in L^{2}\left(\mathbb{R}^{N}\right) .
$$

Since the inclusion $L^{2}\left(\mathbb{R}^{N}\right) \subset H^{-1}\left(\mathbb{R}^{N}\right)$ is dense, we can extend the operator $R$ by continuity, and in this way we get a continuous operator $R_{1}: H^{-1}\left(\mathbb{R}^{N}\right) \rightarrow H^{1}\left(\mathbb{R}^{N}\right)$. If we can show that $R_{1}=(N-\lambda \mathbb{I})^{-1}$, we are done.

We prove this now and we start with the following remark: for each $v \in H^{-1}\left(\mathbb{R}^{N}\right)$, there exists a sequence $\left\{v_{m}\right\}_{m \in \mathbb{N}}$ in $L^{2}\left(\mathbb{R}^{N}\right)$ with $v_{m} \rightarrow v$ in $H^{-1}\left(\mathbb{R}^{N}\right)$ as $m \rightarrow \infty$. Since $R_{1} v_{m} \in H^{2}\left(\mathbb{R}^{N}\right)$, we have that $(N-\lambda \mathbb{I}) R_{1} v_{m}=v_{m}$. Using the continuity of the mapping $(N-\lambda \mathbb{I}) R_{1}: H^{-1}\left(\mathbb{R}^{N}\right) \rightarrow H^{-1}\left(\mathbb{R}^{N}\right)$ we get that

$$
(N-\lambda \mathbb{I}) T_{1} v=v \quad \forall v \in H^{-1}\left(\mathbb{R}^{N}\right),
$$

i.e. $(N-\lambda \mathbb{I}) R_{1}=\mathbb{I}_{H^{-1}\left(\mathbb{R}^{N}\right)}$. A similar argument shows that $R_{1}(N-\lambda \mathbb{I})=\mathbb{I}_{H^{1}\left(\mathbb{R}^{N}\right)}$. Thus, $(N-\lambda \mathbb{I})$ is invertible and $R_{1}=(N-\lambda \mathbb{I})^{-1}$.

STEP 3. The claim now follows from $L_{1}-\lambda \mathbb{I}=N-(\lambda+1) \mathbb{I}$. 
With the help of this preliminary result we can analyse the spectrum of the operator $L_{1}+V$ appearing in the bilinear form

$$
a_{1}(u, v)=a(u, v)+(V u, v)_{L^{2}\left(\mathbb{R}^{N}\right)}=\left\langle\left(L_{1}+V\right) u, v\right\rangle_{H^{-1}\left(\mathbb{R}^{N}\right), H^{1}\left(\mathbb{R}^{N}\right)} .
$$

We have identified here the element $V u \in L^{2}\left(\mathbb{R}^{N}\right)$ with $V u \in H^{-1}\left(\mathbb{R}^{N}\right)$ via

$$
\langle V u, v\rangle_{H^{-1}\left(\mathbb{R}^{N}\right), H^{1}\left(\mathbb{R}^{N}\right)}=(V u, v)_{L^{2}\left(\mathbb{R}^{N}\right)} \quad \forall v \in H^{1}\left(\mathbb{R}^{N}\right) .
$$

We say that $\lambda$ is an eigenvalue of the bounded operator

$$
L_{1}+V: H^{1}\left(\mathbb{R}^{N}\right) \rightarrow H^{-1}\left(\mathbb{R}^{N}\right)
$$

and we write $\lambda \in \sigma_{p}\left(L_{1}+V\right)$ if and only if there exists a $\varphi \in H^{1}\left(\mathbb{R}^{N}\right) \backslash\{0\}$, with $\left(L_{1}+V\right) \varphi=\lambda \varphi$ in $H^{-1}\left(\mathbb{R}^{N}\right)$.

Proposition 3.2. Under assumption $V$, the set $\sigma_{p}\left(L_{1}+V\right)$ coincides with the point spectrum $\sigma_{p}(-\Delta+V)$.

Proof. If $\lambda$ is an eigenvalue, we have that

$$
L_{1} \varphi=\lambda \varphi-V \varphi \in L^{2}\left(\mathbb{R}^{N}\right) .
$$

Hence, $\varphi \in H^{2}\left(\mathbb{R}^{N}\right)$ and $(-\Delta+V) \varphi=\lambda \varphi$ in $L^{2}\left(\mathbb{R}^{N}\right)$. This means that $\lambda \in \sigma_{p}(-\Delta+$ $V)$. Thus, we get the desired result.

Proposition 3.3. Suppose that assumption L holds and assume that $\lambda \in]-\infty, 0[\backslash$ $\sigma_{d}(-\Delta+V)$. Then,

$$
\left(L_{1}+V-\lambda \mathbb{I}\right)^{-1}: H^{-1}\left(\mathbb{R}^{N}\right) \rightarrow H^{1}\left(\mathbb{R}^{N}\right)
$$

exists as a bounded operator.

Proof. If $\lambda \in]-\infty, 0\left[\backslash \sigma_{d}(-\Delta+V)\right.$, then

$$
(-\Delta+V-\lambda \mathbb{I})^{-1}: L^{2}\left(\mathbb{R}^{N}\right) \rightarrow L^{2}\left(\mathbb{R}^{N}\right)
$$

exists as a bounded operator.

STEP 1. As a first step, we show that there exists a strictly positive constant $C$ with

$$
\left\|(-\Delta+V-\lambda \mathbb{I})^{-1} u\right\|_{H^{1}\left(\mathbb{R}^{N}\right)} \leqslant C\|u\|_{H}^{-1}\left(\mathbb{R}^{N}\right) \quad \forall u \in L^{2}\left(\mathbb{R}^{N}\right),
$$

i.e. with

$$
\|w\|_{H^{1}\left(\mathbb{R}^{N}\right)} \leqslant C\|(-\Delta+V-\lambda \mathbb{I}) w\|_{H}^{-1}\left(\mathbb{R}^{N}\right) \quad \forall w \in H^{2}\left(\mathbb{R}^{N}\right) .
$$

Suppose, indeed, on the contrary, that there exists some sequence $\left\{w_{m}\right\}_{m \in \mathbb{N}}$ in $H^{2}\left(\mathbb{R}^{N}\right)$ with $\left\|w_{m}\right\|_{H^{1}\left(\mathbb{R}^{N}\right)}=1$, for all $m \in \mathbb{N}$, and $(-\Delta+V-\lambda \mathbb{I}) w_{m} \rightarrow 0$ in $H^{-1}\left(\mathbb{R}^{N}\right)$ as $m \rightarrow \infty$. This would imply that $w_{m} \rightarrow 0$ in $L^{2}\left(\mathbb{R}^{N}\right)$ as $m \rightarrow \infty$. Indeed, for all $\varphi \in H^{2}\left(\mathbb{R}^{N}\right)$, we have that

$$
\begin{aligned}
\left(w_{m},(-\Delta+V-\lambda \mathbb{I}) \varphi\right)_{L^{2}\left(\mathbb{R}^{N}\right)} & =\left((-\Delta+V-\lambda \mathbb{I}) w_{m}, \varphi\right)_{L^{2}\left(\mathbb{R}^{N}\right)} \\
& =\left\langle(-\Delta+V-\lambda \mathbb{I}) w_{m}, \varphi\right\rangle_{H^{-1}\left(\mathbb{R}^{N}\right), H^{1}\left(\mathbb{R}^{N}\right)} \\
& \rightarrow 0
\end{aligned}
$$


as $m \rightarrow \infty$. Since the range of $(-\Delta+V-\lambda I)$ is $L^{2}\left(\mathbb{R}^{N}\right)$, it follows that $w_{m} \rightarrow 0$ in $L^{2}\left(\mathbb{R}^{N}\right)$ as $m \rightarrow \infty$.

Our assumptions on the sequence $\left\{w_{m}\right\}_{m \in \mathbb{N}}$ imply that

$$
\left\langle(-\Delta+V-\lambda \mathbb{I}) w_{m}, w_{m}\right\rangle_{H^{-1}\left(\mathbb{R}^{N}\right), H^{1}\left(\mathbb{R}^{N}\right)} \rightarrow 0 \quad \text { as } m \rightarrow \infty .
$$

This in turn means that

$$
\lim _{m \rightarrow \infty}\left\|\nabla w_{m}\right\|_{L^{2}\left(\mathbb{R}^{N}\right)}^{2}-\lambda\left|w_{m}\right|_{L^{2}\left(\mathbb{R}^{N}\right)}^{2}+\left(V w_{m}, w_{m}\right)_{L^{2}\left(\mathbb{R}^{N}\right)}=0 .
$$

By the equivalence of norms, and since $\lambda<0$, there exists some constant $c_{1}$ with

$$
\liminf _{m \rightarrow \infty}\left\|\nabla w_{m}\right\|_{L^{2}\left(\mathbb{R}^{N}\right)}^{2}-\lambda\left|w_{m}\right|_{L^{2}\left(\mathbb{R}^{N}\right)}^{2} \geqslant c_{1}>0 .
$$

Hence, we get a contradiction if we can show that, up to some subsequence, we have

$$
\lim _{m \rightarrow \infty}\left|\int_{\mathbb{R}^{N}} V w_{m}^{2} \mathrm{~d} x\right| \leqslant \frac{1}{2} c_{1}
$$

But this follows from the complete continuity assumption $\mathrm{V}$ since, up to some subsequence, we may assume that $w_{m}$ converges weakly in $H^{1}\left(\mathbb{R}^{N}\right)$.

SteP 2. We may now extend the operator $(-\Delta+V-\lambda I)^{-1}$ by continuity to a bounded operator $H^{-1}\left(\mathbb{R}^{N}\right) \rightarrow H^{1}\left(\mathbb{R}^{N}\right)$. Using a density argument, as we did in the proof of proposition 3.1, one can show that the so-defined operator is in fact $\left(L_{1}+V-\lambda \mathbb{I}\right)^{-1}$.

\section{Palais-Smale condition}

We intend to solve problem $\mathrm{P}$ with the help of a variational characterization of critical values of $I_{\lambda}$. We will develop these characterizations under a classical compactness assumption called the 'Palais-Smale condition' (see, for example, $[2,6,14]$ ).

Let us consider a Palais-Smale sequence $\left\{u_{m}\right\}_{m \in \mathbb{N}}$ in $H^{1}\left(\mathbb{R}^{N}\right)$. By this we mean a sequence satisfying the conditions

$$
\lim _{m \rightarrow \infty} I_{\lambda}\left(u_{m}\right)=c \in \mathbb{R} \quad \text { and } \quad \lim _{m \rightarrow \infty} I_{\lambda}\left(u_{m}\right)=0 .
$$

Thus, we have that

$$
\begin{aligned}
2 I_{\lambda}\left(u_{m}\right) & =B_{\lambda}\left(u_{m}\right)-2 \Phi\left(u_{m}\right)=2 c+o(1) \\
I_{\lambda}^{\prime}\left(u_{m}\right) u_{m} & =B_{\lambda}\left(u_{m}\right)-(2+\sigma) \Phi\left(u_{m}\right)=o(1)\left\|u_{m}\right\|_{H^{1}\left(\mathbb{R}^{N}\right)} .
\end{aligned}
$$

Eliminating $\Phi\left(u_{m}\right)$ by a linear combination, we get that

$$
2(2+\sigma) c+o(1)+o(1)\left\|u_{m}\right\|_{H^{1}\left(\mathbb{R}^{N}\right)}=\sigma B_{\lambda}\left(u_{m}\right) .
$$

Note that we can replace $\|\cdot\|_{H^{1}\left(\mathbb{R}^{N}\right)}$ in this relation by any other equivalent norm. 
We say that the Palais-Smale condition holds if, up to a subsequence, $\left\{u_{m}\right\}_{m \in \mathbb{N}}$ is a convergent sequence in $H^{1}\left(\mathbb{R}^{N}\right)$. We will now answer the question of whether or not this condition holds. To that end, we will proceed in two steps: first we assume that $\lambda<\lambda_{1}$, and then we proceed with the more difficult case when $\left.\lambda \in\right] \lambda_{1}, 0[$.

\subsection{Palais-Smale sequences when $\lambda<\lambda_{1}$}

Suppose that assumption $\mathrm{V}$ holds. Then, as long as $\lambda$ is strictly below $\lambda_{1}$, we may consider the norm $\|\cdot \cdot\|_{\lambda}$ defined by

$$
\|u\|_{\lambda}:=\sqrt{B_{\lambda}(u)} \quad \forall u \in H^{1}\left(\mathbb{R}^{N}\right) ;
$$

note that this norm is equivalent to the usual norm $\|\cdot\|_{H^{1}\left(\mathbb{R}^{N}\right)}$ in $H^{1}\left(\mathbb{R}^{N}\right)$. We may use this norm in (4.3); this leads us to

$$
\sigma\left\|u_{m}\right\|_{\lambda}^{2}=2(2+\sigma) c+o(1)+o(1)\left\|u_{m}\right\|_{\lambda} .
$$

In this way, we immediately get a first main property exhibited by all Palais-Smale sequences.

Lemma 4.1. Suppose that assumption $L$ holds and let $\lambda<\lambda_{1}$ be kept fixed. Then, every Palais-Smale sequence for $I_{\lambda}$ is bounded in $H^{1}\left(\mathbb{R}^{N}\right)$.

\subsection{Palais-Smale sequences when $\lambda \in] \lambda_{1}, 0[$}

We now consider the situation where $\lambda$ is in a spectral gap, say $\lambda \in] \lambda^{-}, \lambda^{+}[$. Identifying $H^{-1}\left(\mathbb{R}^{N}\right)$ with $H^{1}\left(\mathbb{R}^{N}\right)$, we may consider $L_{1}+V$ as a self-adjoint operator $H^{1}\left(\mathbb{R}^{N}\right) \rightarrow H^{1}\left(\mathbb{R}^{N}\right)$ with

$$
\left(\left(L_{1}+V\right) u, v\right)_{H^{1}\left(\mathbb{R}^{N}\right)}=\int_{\mathbb{R}^{N}} \nabla u \cdot \nabla v+V u v \mathrm{~d} x .
$$

Considered in this way, $L_{1}+V$ has a spectral decomposition of the form

$$
L_{1}+V=\int_{\mathbb{R}} \mu \mathrm{d} E(\mu)=\int_{\lambda_{1}}^{+\infty} \mu \mathrm{d} E(\mu) .
$$

For more details, we refer the reader to Kato [8, pp. 353-356].

We fix some point $\bar{\lambda}$ inside the gap $] \lambda^{-}, \lambda^{+}[$. The operator defined by

$$
P:=\int_{]-\infty, \bar{\lambda}]} \mathrm{d} E(\mu)=E(\bar{\lambda}): H^{1}\left(\mathbb{R}^{N}\right) \rightarrow H^{1}\left(\mathbb{R}^{N}\right)
$$

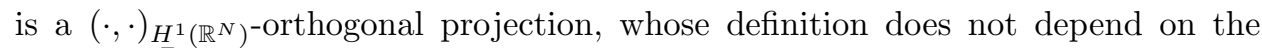
choice of $\bar{\lambda}$. For convenience, we introduce a second $(\cdot, \cdot)_{H^{1}\left(\mathbb{R}^{N}\right)^{-o r t h o g o n a l ~ p r o j e c-~}}$ tion $H^{1}\left(\mathbb{R}^{N}\right) \rightarrow H^{1}\left(\mathbb{R}^{N}\right)$ via $Q:=\mathbb{I}-P$. Note that $P, Q$ and $L_{1}+V$ commute and that the orthogonality implies that $P Q=Q P=0$.

For $\lambda<\lambda_{1}$, we have established the boundedness of Palais-Smale sequences with the help of the well suited norm $\|\cdot\|_{\lambda}$; for $\left.\lambda \in\right] \lambda^{-}, \lambda^{+}$, we again need a norm that is well adapted for questions related to the boundedness of Palais-Smale sequences. 
We denote this norm once more by $\|\cdot \cdot\|_{\lambda}$, and we define it via a new scalar product in the following way:

$$
\begin{aligned}
((u, v))_{\lambda} & =\left(\left(L_{1}+V-\lambda \mathbb{I}\right)(Q-P) u, v\right)_{H^{1}\left(\mathbb{R}^{N}\right)}, \\
\|u\|_{\lambda} & =\sqrt{((u, v))_{\lambda}} .
\end{aligned}
$$

We then get the following proposition.

Proposition 4.2. Suppose that assumption $L$ holds and that $\lambda$ is in a spectral gap ]$\lambda^{-}, \lambda^{+}[$.

Then, \|\|$\cdot \|_{\lambda}$ is a norm on $H^{1}\left(\mathbb{R}^{N}\right)$ that is equivalent to the usual norm on $H^{1}\left(\mathbb{R}^{N}\right)$.

For a proof, we refer the reader to Stuart [19].

With the help of this new norm, we can now deal with the boundedness of PalaisSmale sequences. In fact, the following holds.

Lemma 4.3. Suppose that assumptions $L$ and $Q$ hold and assume that

$$
\left.\lambda_{1} \in\right]-\infty, 0\left[\backslash \sigma_{p}(-\Delta+V) .\right.
$$

Then, every Palais-Smale sequence is bounded in $H^{1}\left(\mathbb{R}^{N}\right)$.

Proof. As we did for $\Phi\left(u_{m}\right)$, we can eliminate $B_{\lambda}\left(u_{m}\right)$ in (4.1) and (4.2). This leads to the relation

$$
\sigma \Phi\left(u_{m}\right)=2 I_{\lambda}\left(u_{m}\right)-I_{\lambda}^{\prime}\left(u_{m}\right) u_{m}=2 c+o(1)+o(1)\left\|u_{m}\right\|_{\lambda} .
$$

We now proceed with an idea found in [9]. Under assumption $Q$ we have, for $|u| \leqslant 1$, that

$$
\left.\left.|q(x)| u\right|^{\sigma} u\right|^{2}=q(x)^{2}|u|^{2+2 \sigma} \leqslant \text { const. } q(x)|u|^{2+\sigma} ;
$$

for $|u| \geqslant 1$, the corresponding relation is

$$
\left.\left.|q(x)| u\right|^{\sigma} u\right|^{(2+\sigma) /(1+\sigma)} \leqslant \text { const. } q(x)|u|^{2+\sigma} .
$$

Setting $\Omega_{m}=\left\{x \in \mathbb{R}^{N}|| u_{m}(x) \mid \leqslant 1\right\}$, we get the estimates

$$
\begin{aligned}
a_{1} & :=\left[\left.\left.\int_{\Omega_{m}}|q(x)| u_{m}(x)\right|^{\sigma} u_{m}(x)\right|^{2}\right]^{1 / 2} \\
& \leqslant \text { const. }\left(2 c+o(1)+o(1)\left\|u_{m}\right\|_{\lambda}\right)^{1 / 2}
\end{aligned}
$$

and

$$
\begin{aligned}
a_{2} & :=\left[\left.\left.\int_{\mathbb{R}^{N} \backslash \Omega_{m}}|q(x)| u_{m}(x)\right|^{\sigma} u_{m}(x)\right|^{(2+\sigma) /(1+\sigma)}\right]^{(1+\sigma) /(2+\sigma)} \\
& \leqslant \text { const. }\left(2 c+o(1)+o(1)\left\||| u_{m}\right\| \|_{\lambda}\right)^{(1+\sigma) /(2+\sigma)}
\end{aligned}
$$


Now, we use the relation $I_{\lambda}^{\prime}\left(u_{m}\right) P u_{m}=\left\|P u_{m}\right\|_{\lambda}^{2}-\Phi^{\prime}\left(u_{m}\right) P u_{m}$. With the help of Hölder's inequality, we get that

$$
\begin{aligned}
\left\|P u_{m}\right\|_{\lambda}^{2} & =-I_{\lambda}^{\prime}\left(u_{m}\right) P u_{m}-\int_{\mathbb{R}^{N}} q\left|u_{m}\right|^{\sigma} u_{m} P u_{m} \mathrm{~d} x \\
& \leqslant\left. o(1)|| P u_{m}\left|\|_{\lambda}+a_{1}\right| P u_{m}\right|_{L^{2}\left(\mathbb{R}^{N}\right)}+a_{2}\left|P u_{m}\right|_{L^{2+\sigma}\left(\mathbb{R}^{N}\right)} \\
& \leqslant\left[o(1)+\text { const. } a_{1}+\text { const. } a_{2}\right]\left|\left\|P u_{m}|\||_{\lambda}\right.\right. \\
\left\|P u_{m}\right\|_{\lambda} & \leqslant o(1)+\text { const. } a_{1}+\text { const. } a_{2} .
\end{aligned}
$$

In a similar way, one can derive the estimate

$$
\left\|Q u_{m}\right\|_{\lambda} \leqslant o(1)+\text { const. } a_{1}+\text { const. } a_{2} .
$$

We recall now that $P Q=0$; combining this with the above result, we can write

$$
\left\|u_{m}\right\|_{\lambda}^{2}=\left\|P u_{m}\right\|_{\lambda}^{2}+\left\|Q u_{m}\right\|_{\lambda}^{2} \leqslant\left[o(1)+\text { const. } a_{1}+\text { const. } a_{2}\right]^{2} .
$$

Using the estimates derived above for $a_{1}$ and $a_{2}$, we get that

$$
\begin{aligned}
\left\|u_{m} \mid\right\|_{\lambda}^{2} \leqslant 2[o(1)+\text { const. }( & \left.2 c+o(1)+o(1)\left\|u_{m} \mid\right\|_{\lambda}\right)^{1 / 2} \\
& \left.+ \text { const. }\left(2 c+o(1)+o(1)\|\| u_{m} \|_{\lambda}\right)^{(1+\sigma) /(2+\sigma)}\right]^{2} .
\end{aligned}
$$

This shows that $\left\|\left|u_{m}\right|\right\|_{\lambda}$ is bounded. Hence, every Palais-Smale sequence is bounded with respect to all norms that are equivalent to $\left\|u_{m}\right\| \|_{\lambda}$.

\subsection{The convergence of Palais-Smale sequences}

Combining the results of lemmata 4.1 and 4.3 , we affirm that every Palais-Smale sequence is bounded. We can even strengthen this result in the following way.

Proposition 4.4. Suppose that assumptions $L$ and $Q$ hold, and that $\lambda \in]-\infty, 0[\backslash$ $\sigma_{p}(-\Delta+V)$.

Then, every Palais-Smale sequence converges up to some subsequence.

Proof. Due to the boundedness of all Palais-Smale sequences, we may assume that, up to some subsequence, every Palais-Smale sequence $\left\{u_{m}\right\}_{m \in \mathbb{N}}$ is weakly convergent. Thus, we have that $u_{m} \rightarrow u$ in $H^{1}\left(\mathbb{R}^{N}\right)$ and $\Phi^{\prime}\left(u_{m}\right) \rightarrow \Phi^{\prime}(u)$ in $H^{-1}\left(\mathbb{R}^{N}\right)$ as $m \rightarrow \infty$. But, this implies that

$$
\left(L_{1}+V-\lambda \mathbb{I}\right) u_{m}=I_{\lambda}^{\prime}\left(u_{m}\right)+\Phi^{\prime}\left(u_{m}\right) \rightarrow \Phi^{\prime}(u) \text { in } H^{-1}\left(\mathbb{R}^{N}\right) .
$$

Hence, since $\lambda \notin \sigma_{p}(-\Delta+V)$, we may conclude that $u_{m} \rightarrow u$ in $H^{1}\left(\mathbb{R}^{N}\right)$ as $m \rightarrow \infty$, and this is the desired result.

\section{Critical point theory and bifurcation theory}

In this section, we develop a critical point theory and a bifurcation theory within the following context.

Condition $\mathrm{C}_{0}$. We denote by $X$ a separable, real Hilbert space equipped with a scalar product $(\cdot \mid \cdot)$ and a corresponding norm $\|\cdot\|$. This Hilbert space is split into 
an orthogonal sum $X=Y \oplus Z$. In this decomposition, $Y$ is a finite-dimensional space of dimension $n$; we thereby explicitly do not exclude the situation where $Y=\{0\}$ and $n=0$.

Inside the subspace $Z$, we consider an orthonormal set $\left\{z_{1}, z_{2}, \ldots, z_{m}\right\}(m \geqslant 1)$ as well as a 'circle' $S_{n}:=\{z \in Z \mid\|z\|=\rho\}$ of radius $\rho>0$.

For any $R>\rho$, we collect in the set $\overline{\mathcal{A}}_{n, m}$ all the points $x \in X$ of the form

$$
y+\sum_{k=1}^{m} \alpha_{k} z_{k}
$$

where $y \in Y, \alpha_{k} \in \mathbb{R}$ for $k=1, \ldots, m$ and $\|x\| \leqslant R$. We set $\mathcal{A}_{n, m}:=\left\{x \in \overline{\mathcal{A}}_{n, m} \mid\right.$ $0<\|x\|<R\}$ and $\partial \mathcal{A}_{n, m}:=\overline{\mathcal{A}}_{n, m} \backslash \mathcal{A}_{n, m}$.

As well as these sets, we consider the class $\Gamma_{n, m}$ consisting of all odd homeomorphisms $\gamma: X \rightarrow X$, where $\left.\gamma\right|_{\partial \mathcal{A}_{n, m}}$ is the identity map on $\partial \mathcal{A}_{n, m}$.

\subsection{Odd linking}

Within context $\mathrm{C}_{0}$ defined above, we say that $S_{n}$ links $\mathcal{A}_{n, m}$ oddly if $\gamma\left(\mathcal{A}_{n, m}\right) \cap$ $S_{n} \neq \emptyset$ for all homeomorphisms $\gamma \in \Gamma_{n, m}$. Odd linking will play a key role when we characterize critical levels of energy functionals.

The following proposition shows that the size of $\gamma\left(\mathcal{A}_{n, m}\right) \cap S_{n}$ can be characterized by its genus. We refer the reader to Struwe [14] or Chow and Hale [2] for more details about the genus of an odd set.

Proposition 5.1. Within the context $C_{0}$, the set $\gamma^{-1}\left(S_{n}\right) \cap \mathcal{A}_{n, m}$ is of genus $\geqslant m$ for all homeomorphisms $\gamma \in \Gamma_{n, m}$. Hence, $S_{n}$ links $\mathcal{A}_{n, m}$ oddly.

Proof. We denote by $B$ the open ball of radius $\rho$ in $X$, and consider a homeomorphism $\gamma \in \Gamma_{n, m}$. Since $0<\rho<R$, we get that $\gamma^{-1}(B) \cap \partial \mathcal{A}_{n, m}=\{0\}$. Moreover, the set $U:=\gamma^{-1}(B) \cap \operatorname{span}\left\{\mathcal{A}_{n, m}\right\}$ is an open set relative to a subspace of dimension $n+m$. Thus, $\partial U=\gamma^{-1}(\partial B) \cap \operatorname{span}\left\{\mathcal{A}_{n, m}\right\}$ is of genus $n+m$.

We set $K:=\gamma^{-1}\left(S_{n}\right) \cap \operatorname{span}\left\{\mathcal{A}_{n, m}\right\}$ and we note that $K \subset \mathcal{A}_{n, m}$. Thus, it is enough to show that the genus of $K$ is at least $m$.

If $n=0$, we have that $\partial B=S_{0}$, so $K$ is of genus $m$.

If $n \geqslant 1$, we can argue as follows. We consider, for $k=1, \ldots, n$, the sets $V_{k}:=$ $\gamma^{-1}\left(\left\{x \in B \mid\left(x \mid y_{k}\right) \neq 0\right\}\right)$; all these sets are open, odd and oddly contractible. We now assume, on the contrary, that the genus $g$ of $K$ is smaller than $m$. Then, there exist open and odd sets $W_{1}, \ldots, W_{g}$ that are oddly contractible, with $K \subset \bigcup_{i=1}^{g} W_{i}$. Hence, we get that

$$
\partial U \subset \bigcup_{k=1}^{n} V_{k} \cup \bigcup_{i=1}^{g} W_{i} .
$$

This is the desired contraction, since this inclusion means that the genus of $\partial U$ is at most $n+g$, a number that is strictly smaller than $n+m$.

\subsection{Existence of critical points}

We now consider a $C^{1}$-functional $I: X \rightarrow \mathbb{R}$ defined in the context $\mathrm{C}_{0}$ and satisfying the following conditions. 
Condition $\mathrm{C}_{1}$. $I$ is even and there exists a constant $\alpha>0$ such that $\left.I\right|_{S_{n}} \geqslant \alpha$.

Condition $\mathrm{C}_{2} .\left.I\right|_{\partial \mathcal{A}_{n, m}} \leqslant 0$.

Condition $\mathrm{C}_{3}$. I satisfies the Palais-Smale condition for all positive levels $c$.

These assumptions imply that the functional $I$ possesses a generalized mountain pass geometry. Ambrosetti and Rabinowitz [1] have studied the existence of critical points for such functionals. We choose another approach and we propose a new variational characterization of critical levels. Note that our method extends to situations where $\operatorname{dim} Y=\infty$, and we will discuss such extensions in a forthcoming article [13].

We start with the candidate

$$
c_{n, m}:=\inf _{\gamma \in \Gamma_{n, m}} \max _{u \in \overline{\mathcal{A}}_{n, m}} I(\gamma(u)) .
$$

Note that proposition 5.1 immediately implies that $c_{n, m} \geqslant \alpha>0$. The following proposition justifies our interest in $c_{n, m}$.

Proposition 5.2. Suppose that $I: X \rightarrow \mathbb{R}$ is a functional operating in the context $C_{0}$ (for some $m \geqslant 1$ ) and exhibiting the properties $C_{1}-C_{3}$.

Then, $c_{n, m}=\inf _{\gamma \in \Gamma_{n, m}} \max _{u \in \overline{\mathcal{A}}_{n, m}} I(\gamma(u))$ is a critical value of $I$.

Proof. Suppose, on the contrary, that $c_{n, m}$ is a regular value. For any given $\varepsilon \in$ ] $0, c_{n, m} / 2$ [, we may then choose a mapping $\gamma \in \Gamma_{n, m}$ in such a way that

$$
\max _{u \in \overline{\mathcal{A}}_{n, m}} I(\gamma(u)) \in\left[c_{n, m}, c_{n, m}+\varepsilon\right] .
$$

By the classical deformation theorem (see, for example, Struwe [14]), there exists, when $\varepsilon$ is small enough, a deformation $\eta:[0,1] \times X \rightarrow X$ such that $\gamma \circ \eta(1, \cdot) \in \Gamma_{n, m}$ and such that $\max _{u \in \overline{\mathcal{A}}_{n, m}} I(\gamma(\eta(u))) \leqslant c_{n, m}-\varepsilon$. But, this contradicts the definition of $c_{n, m}$.

Note that we can successively choose in $Z$ the orthonormal sets $\left\{z_{1}\right\},\left\{z_{1}, z_{2}\right\}$, $\left\{z_{1}, z_{2}, z_{3}\right\}, \ldots$ In this way, we obtain a sequence of non-decreasing critical values $c_{n, k}$, where the index $k$ takes all the values $k=1,2, \ldots$, with $k \leqslant \operatorname{dim} Z$. Nevertheless, even if $\operatorname{dim} Z>1$, we cannot guarantee the existence of more than one critical point for $I$, since all the values $c_{n, 1}, c_{n, 2}, \ldots$ may coincide. There is a way out if $\operatorname{dim} Z=\infty$ and if one can show, for example, that $\lim _{k \rightarrow \infty} c_{n, k}=+\infty$. We present here an alternative based on additional critical values.

For $k=1, \ldots, m-1$, we consider the sets

$$
V_{k}:=\{U \subset X \mid U=-U, 0 \notin \bar{U} \text { and } U \text { is of genus } \leqslant k\} .
$$

For convenience, we set $V_{0}:=\{\emptyset\}$.

We introduce a new set of candidates

$$
d_{n, m, k}:=\inf _{\gamma \in \Gamma_{n, m}} \inf _{U \in V_{k}} \max _{\mathcal{\mathcal { A }}_{n, m} \backslash U} I(\gamma(u)) \text { for } k=0, \ldots, m-1 .
$$

At this point, we must make the following remarks. 
- Obviously, $d_{n, m, 0}=c_{n, m}$.

- For all $\gamma \in \Gamma_{n, m}$, the intersection $\gamma^{-1}\left(S_{n}\right) \cap \mathcal{A}_{n, m}$ is of genus $\geqslant m$; hence, $d_{n, m, k} \geqslant \alpha>0$ for $k=0,1,2, \ldots, m-1$.

- We have $\alpha \leqslant d_{n, m, m-1} \leqslant d_{n, m, m-2} \leqslant \cdots \leqslant d_{n, m, 0}=c_{n, m}$.

Proceeding as in the proof of proposition 5.2, we get the following.

Proposition 5.3. In the context $C_{0}$, the values

$$
d_{n, m, k}:=\inf _{\gamma \in \Gamma_{n, m}} \inf _{U \in V_{k}} \max _{\overline{\mathcal{A}}_{n, m} \backslash U} I(\gamma(u)),
$$

where $k=0,1,2, \ldots, m-1$, are critical values of I provided this functional I exhibits the properties $C_{1}-C_{3}$.

Even if we have associated with each critical value $c_{n, m}$ a strictly positive, nonincreasing sequence of critical values $d_{n, m, k}, k=0,1,2, \ldots, m-1$, the possible coincidence of these values prohibits at first sight the formulation of a multiplicity result. But looking somewhat closer, we can obtain a multiplicity result.

THEOREM 5.4 (existence of multiple critical points). Suppose that the functional $I: X=Y \oplus Z \rightarrow \mathbb{R}$ operates for some $m \geqslant 1$ in the context $C_{0}$ and has the properties $C_{1}-C_{3}$.

Then, there exist at least $m$ pairs $\pm u \neq 0$ of critical points of I corresponding to the level sets

$$
\begin{aligned}
& d_{n, m, 0}=c_{n, m}=\inf _{\gamma \in \Gamma_{n, m}} \max _{u \in \overline{\mathcal{A}}_{n, m}} J(\gamma(u)), \\
& d_{n, m, k}=\inf _{\gamma \in \Gamma_{n, m}} \inf _{V \in V_{k}} \max _{u \in \overline{\mathcal{A}}_{n, m} \backslash V} J(\gamma(u)), \quad k=1,2 \ldots, m-1,
\end{aligned}
$$

where the sets $V_{k}$ are defined in (5.1).

If, moreover, $d_{n, m, k}=d_{n, m, k+1}=\cdots=d_{n, m, k+s}$ for some $k \in\{0,1,2, \ldots, m-$ $s-1\}$ and $s \in\{1,2, \ldots, m-1\}$, then the number of critical points corresponding to this common value is a set of genus $\geqslant s+1$. Thus, the functional I possesses an infinite number of critical points corresponding to this common critical value.

Proof. Suppose, indeed, that $d_{n, m, k}=d_{n, m, k+1}$ for some $k \in\{0,1,2, \ldots, m-$ $2\}$. Furthermore, suppose that there exists only one pair $\pm u_{1}$ of critical points corresponding to this common value $d_{n, m, k}=d_{n, m, k+1}$. Under these circumstances, we choose small, disjoint neighbourhoods $\pm U_{1}$ of $\pm u_{1}$ in such a way that $U_{1} \cup\left(-U_{1}\right)$ is oddly contractible.

Given any (small) $\varepsilon>0$, there exist a homeomorphism $\gamma \in \Gamma_{n, m}$ and a set $V \in V_{k}$ such that $\max _{u \in \overline{\mathcal{A}}_{n, m} \backslash V} I(\gamma(u))<d_{n, m, k}+\varepsilon$. Applying the classical deformation theorem, we get a mapping $\eta:[0,1] \times X \rightarrow X$ such that $\gamma \circ \eta(1, \cdot) \in \Gamma_{n, m}$. We set $U:=\eta^{-1}\left(1, U_{1}\right)$, and we note that $V \cup \pm U$ belongs to $V_{k+1}$. In this way, we get the desired contradiction

$$
\max _{u \in \overline{\mathcal{A}}_{n, m} \backslash(V \cup \pm U)} I(\gamma(\eta(1, u))) \leqslant d_{n, m, k+1}-\varepsilon=d_{n, m, k}-\varepsilon .
$$


A detailed analysis of this proof shows that we indeed have a 'classical multiplicity result': if $d_{n, m, k}=d_{n, m, k+1}$, the corresponding set of critical points is of at least genus 2 , if $d_{n, m, k}=d_{n, m, k+1}=d_{n, m, k+2}$, the corresponding set of critical points is of at least genus 3 , and so forth.

\subsection{Bifurcation of the level sets $d_{n, m, k}$}

We now extend the setting, and we assume that the functional $I$ depends on some real parameter $\lambda$. Thus, we denote the functional by $I_{\lambda}$ instead of $I$. Thereby, the parameter $\lambda$ varies inside an open interval $] \lambda^{-}, \lambda^{+}$. We analyse the bifurcation behaviour of the level set $d_{n, m, k}(\lambda)$; here, again, we exhibit the dependence on the parameter $\lambda$.

We follow an idea inspired by [4] and we will work under the following assumptions on $I_{\lambda}$.

Assumption $\mathrm{B}_{1}$. For each fixed value of $u \in X$, the functional $I_{\lambda}(u)$ is non-increasing in $\lambda$.

Assumption $\mathrm{B}_{2}$. There exists a constant $\tau>1$ such that, for $k=0,1, \ldots, m-1$, we have that

$$
0<d_{n, m, k}(\lambda) \leqslant \text { const. }\left(\lambda^{+}-\lambda\right)^{\tau}
$$

as $\lambda \rightarrow \lambda^{+}$, with $\lambda<\lambda^{+}$.

Assumption $B_{3}$. For all $\lambda^{(1)}$ and $\lambda^{(2)}$, with $\lambda^{-}<\lambda^{(1)}<\lambda^{(2)}<\lambda^{+}$, we have that

$$
I_{\lambda^{(1)}}(v)-I_{\lambda^{(2)}}(v) \geqslant \text { const. }\left(\lambda^{(2)}-\lambda^{(1)}\right) \| v \mid \quad \forall v \in X,
$$

where $|\cdot|$ is some norm on $X$, with $|u| \leqslant\|u\|$, for all $u \in X$; thereby, $X$ must not necessarily be complete with respect to the norm $|\cdot|$.

REMARK 5.5. Under assumption $\mathrm{B}_{1}$, we fix some $\Lambda$ in $] \lambda^{-}, \lambda^{+}$. Then, we can choose the set $\overline{\mathcal{A}}_{n, m}$, as well as $\Gamma_{n, m}$, in a fixed way for all $\lambda \in\left[\Lambda, \lambda^{+}\right.$[. The critical values $d_{n, m, k}(\lambda)$ obtained in such a way are non-increasing in $\lambda$.

The monotonicity of $I_{\lambda}$ in (B2) implies that the derivative $d_{n, m, k}^{\prime}(\lambda)$ exists almost everywhere (a.e.) on $] \lambda^{-}, \lambda^{+}$[ and, whenever this derivative exists, we have that $d_{n, m, k}^{\prime}(\lambda) \leqslant 0$. We can even derive a somewhat stronger result, as in the following.

Proposition 5.6. Under assumptions $C_{0}-C_{3}$ and $B_{1}-B_{3}$, there exists a sequence of points $\left\{\lambda^{(j)}\right\}_{j \in \mathbb{N}}$ with the following properties.

(1) $\lambda^{(j)}<\lambda^{+}$for all $j \in \mathbb{N}$.

(2) $\lambda^{(j)} \rightarrow \lambda^{+}$as $j \rightarrow \infty$.

(3) The derivatives

$$
d_{n, m, 0}^{\prime}\left(\lambda^{(j)}\right), d_{n, m, 1}^{\prime}\left(\lambda^{(j)}\right), \ldots, d_{n, m, m-1}^{\prime}\left(\lambda^{(j)}\right)
$$

all exist and, for $k=0,1, \ldots, m-1, d_{n, m, k}^{\prime}\left(\lambda^{(j)}\right) \rightarrow 0$ as $j \rightarrow \infty$. 
Proof. Otherwise, we consider the limit

$$
a_{0}:=\liminf \left(-\sum_{k=0}^{m-1} d_{n, m, k}^{\prime}(\lambda)\right)>0 ;
$$

we compute this limit for $\lambda \rightarrow \lambda^{+}$and $\lambda<\lambda^{+}$along points where all $d_{n, m, k}^{\prime}(\lambda)$ exist. Thus, for $\lambda<\lambda^{+}$, we have that

$$
\begin{aligned}
\sum_{k=0}^{m-1} d_{n, m, k}(\lambda) & =\sum_{k=0}^{m-1} d_{n, m, k}(\lambda)-\lim _{s \rightarrow \lambda^{+}, s<\lambda^{+}} \sum_{k=0}^{m-1} d_{n, m, k}(s) \\
& \geqslant \lim _{s \rightarrow \lambda^{+}, s<\lambda^{+}} \int_{\lambda}^{s}\left[-\sum_{k=0}^{m-1} d_{n, m, k}^{\prime}(t) \mathrm{d} t\right] \\
& \geqslant \frac{1}{2} a_{0}\left(\lambda^{+}-\lambda\right) .
\end{aligned}
$$

This leads us to the desired contradiction

$$
\frac{1}{2} a_{0}\left(\lambda^{+}-\lambda\right) \leqslant \sum_{k=0}^{m-1} d_{n, m, k}(\lambda) \leqslant \text { const. }\left(\lambda^{+}-\lambda\right)^{\tau} \quad \text { as } \lambda \rightarrow \lambda^{+} \text {(from the left). }
$$

We now define what we mean by a bifurcation point within the context $\mathrm{C}_{0}-\mathrm{C}_{3}$ and $\mathrm{B}_{1}-\mathrm{B}_{3}$.

1. We say that $\lambda^{+}$is a $|\cdot|$-bifurcation point if there exists a sequence $\left\{\lambda^{(j)}\right\}_{j \in \mathbb{N}}$ in $] \lambda^{-}, \lambda^{+}$[ with the following properties.

(a) For each $j \in \mathbb{N}$, there exists a $u^{(j)}$ in $X \backslash\{0\}$, with $I_{\lambda^{(j)}}^{\prime}\left(u^{(j)}\right)=0$.

(b) $\lim _{j \rightarrow \infty} \lambda^{(j)}=\lambda^{+}$and $\lim _{j \rightarrow \infty}\left|u^{(j)}\right|=0$.

2. We say that $\lambda^{+}$is a $|\cdot|$-bifurcation point of multiplicity $m(m>1)$ if there exists a sequence $\left\{\lambda^{(j)}\right\}_{j \in \mathbb{N}}$ in $] \lambda^{-}, \lambda^{+}$[ with the following properties.

(a) For each $j \in \mathbb{N}$, there exists a collection of $m$ different pairs of points $\pm u_{1}^{(j)}, \ldots, \pm u_{m}^{(j)}$ in $X \backslash\{0\}$, with $I_{\lambda^{(j)}}^{\prime}\left(u_{i}^{(j)}\right)=0$ for $i=1, \ldots, m$.

(b) $\lim _{j \rightarrow \infty} \lambda^{(j)}=\lambda^{+}$and $\lim _{j \rightarrow \infty}\left|u_{i}^{(j)}\right|=0$ for $i=1, \ldots, m$.

We can now formulate a first bifurcation result.

TheOREm 5.7. Suppose that $I_{\lambda}$ satisfies, for some $m \geqslant 1$, all the assumptions $C_{0^{-}}$ $C_{3}$ and $B_{1}-B_{3}$.

Then, $\lambda^{+}$is a $|\cdot|$-bifurcation point for $I_{\lambda}^{\prime}(u)=0$.

Proof. Consider some $\lambda \in] \lambda^{-}, \lambda^{+}\left[\right.$, where the derivative $d_{n, m, k}^{\prime}(\lambda)$ exists for some $k \in\{0,1, \ldots, m-1\}$. By proposition 5.2 , the set

$$
K:=\left\{u \in H^{1}\left(\mathbb{R}^{N}\right) \mid I_{\lambda}^{\prime}(u)=0, I_{\lambda}(u)=d_{n, m, k}(\lambda)\right\}
$$

is non-empty. We set $\alpha(\lambda):=\left(\lambda-\lambda^{+}\right)^{2}$, and we consider, for $u \in K$, the open balls $U(u)=\{v \in X \mid\|v-u\|<\alpha(u, \lambda)\}$, with $\alpha(u, \lambda)=\min \left\{\frac{1}{2}\|u\|, \alpha(\lambda)\right\}$. Due to the 
Palais-Smale condition, $K$ is compact. We may thus extract a finite subcover, say $\left\{U\left(u_{i}\right)\right\}_{i=1, \ldots, p}$, such that $K \subset U:=\bigcup_{i=1}^{p} U\left(u_{i}\right)$.

By the deformation theorem (see Struwe [14]), there exist some $\varepsilon \in] 0, \frac{1}{2} d_{n, m, k}(\lambda)[$ and a continuous 1 -parameter family of homeomorphisms $\eta:[0,+\infty[\times X \rightarrow X$ having the following properties.

- $\eta(t, \cdot): X \rightarrow X$ is an odd homeomorphism, with $\eta(t, u)=u$ if $t=0$ or $I_{\lambda}^{\prime}(u)=0$ or $u \notin I_{\lambda}^{-1}\left(\left[d_{n, m, k}(\lambda) / 2,3 d_{n, m, k}(\lambda) / 2\right]\right)$.

- $I_{\lambda}(\eta(t, u))$ is non-increasing in $t$ for all $u \in X$ kept fixed.

- $I_{\lambda}(\eta(t, u))<d_{n, m, k}(\lambda)-\varepsilon$ if $u \in I_{\lambda}^{-1}(]-\infty, d_{n, m, k}(\lambda)+\varepsilon[\backslash U)$.

- If $u \in I_{\lambda}^{-1}(]-\infty, d_{n, m, k}(\lambda)+\varepsilon[)$ and $I_{\lambda}(\eta(1, u)) \geqslant d_{n, m, k}(\lambda)-\varepsilon$, then $\eta(1, u) \in$ $U$.

We now choose $\delta>0$ so small that $d_{n, m, k}(\lambda-\delta)+\delta^{2}<d_{n, m, k}(\lambda)+\varepsilon$. Such a choice of $\delta$ is possible since the derivative $d_{n, m, k}^{\prime}(\lambda)$ exists, and thus

$$
\lim _{\delta \rightarrow 0^{+}} d_{n, m, k}(\lambda-\delta)=d_{n, m, k}(\lambda) .
$$

Note that $\delta^{2}<\varepsilon$ and that, once we have fixed such a $\delta>0$, we may use any other positive value below $\delta$ as a replacement for $\delta$.

We consider the set $D_{k}$ consisting of all $u \in X$ satisfying both $d_{n, m, k}(\lambda)-\delta^{2} \leqslant$ $I_{\lambda}(u)$ and $I_{\lambda-\delta}(u) \leqslant d_{n, m, k}(\lambda-\delta)+\delta^{2}$. Note that this set is non-empty, since we can determine a point belonging to it in the following way. We choose $\gamma \in \Gamma_{n, m}$ and $V \in V_{k}$ with

$$
\max _{u \in \overline{\mathcal{A}}_{n, m} \backslash V} I_{\lambda-\delta}(\gamma(u)) \leqslant d_{n, m, k}(\lambda-\delta)+\delta^{2} .
$$

Using the monotone dependence of $I_{\lambda}$ on $\lambda$, we even get that

$$
d_{n, m, k}(\lambda) \leqslant \max _{u \in \overline{\mathcal{A}}_{n, m} \backslash V} I_{\lambda}(\gamma(u)) \leqslant d_{n, m, k}(\lambda-\delta)+\delta^{2}<d_{n, m, k}(\lambda)+\varepsilon .
$$

This maximum is achieved at some point $\bar{u} \in \overline{\mathcal{A}}_{n, m} \backslash V$; the point $\gamma(\bar{u})$ belongs to $D_{k}$.

Note that we have that $D_{k} \cap U \neq \emptyset$, for otherwise we would get the contradiction

$$
\max _{u \in \overline{\mathcal{A}}_{n, m} \backslash V} I_{\lambda}(\eta(1, \gamma(u))) \leqslant d_{n, m, k}(\lambda)-\varepsilon,
$$

with $\eta(1, \gamma(\cdot)) \in \Gamma_{n, m}$.

Hence, we may choose an element $v \in D_{k} \cap U$. For such an element, we obtain that

$$
\begin{aligned}
\text { const. }|v|^{2} & \leqslant \frac{I_{\lambda-\delta}(v)-I_{\lambda}(v)}{\delta} \\
& \leqslant \frac{d_{n, m, k}(\lambda-\delta)+\delta^{2}-d_{n, m, k}(\lambda)+\delta^{2}}{\delta} \\
& =-\underbrace{\frac{d_{n, m, k}(\lambda-\delta)-d_{n, m, k}(\lambda)}{-\delta}}_{=d_{n, m, k}^{\prime}(\lambda)+o(1) \text { as } \delta \rightarrow 0^{+}}+2 \delta .
\end{aligned}
$$


If $\delta>0$ is small enough, this implies that $|v|^{2} \leqslant-$ const. $d_{n, m, k}^{\prime}(\lambda)+$ const. $\alpha(\lambda)$. Thus, there exists some $u^{(\lambda)} \in K$ with

$$
\left|u^{(\lambda)}\right|^{2} \leqslant- \text { const. } d_{n, m, k}^{\prime}(\lambda)+\text { const. } \alpha(\lambda)+\alpha(\lambda)^{2} .
$$

Applying this estimate to the sequence $\left\{\lambda^{(j)}\right\}_{j \in \mathbb{N}}$ of proposition 5.6, we get the desired result, with $u^{(j)}:=u^{\left(\lambda_{j}\right)}$.

We now address the question of multiple bifurcation. Thus, we assume that $\mathrm{C}_{0^{-}}$ $\mathrm{C}_{3}$ and $\mathrm{B}_{1}-\mathrm{B}_{3}$ hold, with $m \geqslant 2$. We consider the sequence $\left\{\lambda^{(j)}\right\}_{j \in \mathbb{N}}$ given in proposition 5.6. If, up to a subsequence, we have

$$
d_{n, m, m-1}\left(\lambda^{(j)}\right)<d_{n, m, m-2}\left(\lambda^{(j)}\right)<\cdots<d_{n, m, 1}\left(\lambda^{(j)}\right)<d_{n, m, 0}\left(\lambda^{(j)}\right) \quad \forall j \in \mathbb{N},
$$

then the proof of proposition 5.7 implies that $\lambda^{+}$is a $|\cdot|$-bifurcation point of multiplicity $m$ (or higher). This remains true if some of the critical levels coincide.

TheOREm 5.8. Suppose that $I_{\lambda}$ satisfies, for some $m \geqslant 2$, all the assumptions $C_{0^{-}}$ $C_{3}$ and $B_{1}-B_{3}$.

Then, $\lambda^{+}$is a $|\cdot|$-bifurcation point of multiplicity $m$ (at least).

Proof. Suppose that

$$
d_{n, m, k}(\lambda)=d_{n, m, k+1}(\lambda)=\cdots=d_{n, m, k+s}(\lambda)
$$

for some $k \in\{0,1, \ldots, m-2\}$ and for some $s \in\{1,2, \ldots, m-k-1\}$; here $\lambda$ is one of the points of the sequence $\left\{\lambda^{(j)}\right\}_{j \in \mathbb{N}}$ given in proposition 5.6. We then proceed as we did in the proof of theorem 5.7.

The set $\gamma^{-1}\left(D_{k} \cap U\right) \cap \mathcal{A}_{n, m}$ must be of genus $\geqslant s+1$. Since, otherwise, we can derive a contradiction in the following way. First, choose $\gamma \in \Gamma_{n, m}$ and $V \in V_{k}$ such that

$$
\max _{u \in \overline{\mathcal{A}}_{n, m} \backslash V} I_{\lambda-\delta}(\gamma(u)) \leqslant d_{n, m, k}(\lambda-\delta)+\delta^{2} .
$$

Due to $\left(\mathrm{B}_{1}\right)$, we may assert that

$$
d_{n, m, k}(\lambda) \leqslant \max _{u \in \overline{\mathcal{A}}_{n, m} \backslash V} I_{\lambda}(\gamma(u)) \leqslant d_{n, m, k}(\lambda-\delta)+\delta^{2}<d_{n, m, k}(\lambda)+\varepsilon .
$$

Set $\tilde{V}:=V \cup\left(\gamma^{-1}\left(D_{k} \cap U\right) \cap \mathcal{A}_{n, m}\right)$ and note that the genus of $\tilde{V}$ is $\leqslant k+s$. This gives the desired contradiction

$$
d_{n, m, k+s}(\lambda) \leqslant \max _{u \in \overline{\mathcal{A}}_{n, m} \backslash \tilde{V}} I_{\lambda}(\eta(1, \gamma(u)))<d_{n, m, k}(\lambda)-\varepsilon=d_{n, m, k+s}(\lambda)-\varepsilon .
$$

We know that, for all $v \in D_{k} \cap U$, we have that

$$
|v|^{2} \leqslant \text { const. } d_{n, m, k}^{\prime}(\lambda)+\text { const. } \alpha(\lambda)+\alpha(\lambda)^{2} .
$$

Recall that

$$
U=\bigcup_{i=1}^{p} \pm U\left(u_{i}\right)
$$


So we collect in the set $J$ all the indices $i \in\{1, \ldots, p\}$, with $\left(U\left(u_{i}\right) \cup-U\left(u_{i}\right)\right) \cap D_{k} \neq$ $\emptyset$. Note that $J$ must contain at least $s+1$ elements, since the genus of $U \cap D_{k}$ is $\geqslant s+1$. Hence, we get (at least) $s+1$ pairs $\pm u_{i} \in K$, with

$$
\left|u_{i}\right| \leqslant \text { const. } d_{n, m, k}^{\prime}(\lambda)+\text { const. } \alpha(\lambda)+\alpha(\lambda)^{2} .
$$

We can now complete the proof as we did for theorem 5.7.

\section{Existence of multiple solutions for $\lambda<\lambda_{1}$}

We apply the abstract results to the functional $I_{\lambda}=\frac{1}{2} B_{\lambda}(u)-\Phi(u)$ of $\S 2$. We subdivide our analysis into two parts: in this section, we suppose that $\lambda<\lambda_{1}$, and we will treat the case where $\lambda$ lies in a spectral gap in the next section.

\subsection{Existence of a first critical value}

We establish the existence of a first solution pair $\left(\lambda, u_{1, \lambda}\right)$ for problem $\mathrm{P}$, when $\lambda<\lambda_{1}$. We do this under the following additional assumption.

Assumption $\mathrm{E}_{1}$. There exists a one-dimensional subspace $F_{1}$ of $H^{1}\left(\mathbb{R}^{N}\right)$, on which $\Phi$ is essentially positive, i.e. on which we have that $\Phi(w)>0$ for all $w \in F_{1} \backslash\{0\}$. We denote by $w_{1}$ some fixed element in $F_{1}$ with $\left\|w_{1}\right\|_{H^{1}\left(\mathbb{R}^{N}\right)}=1$.

Note that the above assumption is a necessary condition for the existence of (nontrivial) solutions of problem $\mathrm{P}$. Assumption $\mathrm{E}_{1}$ holds if, for example, $q$ is strictly positive on some open set in $\mathbb{R}^{N}$.

THEOREM 6.1. Suppose that assumptions $L, Q$ and $E_{1}$ hold.

Then, for all $\lambda<\lambda_{1}$, there exists (at least) one solution pair $\left(\lambda, \pm u_{1, \lambda}\right)$ of problem $P$.

Proof. We fix some negative value $\Lambda<\lambda_{1}$; we are going to establish the existence of solutions for each $\lambda \in\left[\Lambda, \lambda_{1}[\right.$. This will prove the existence of solutions for all values of $\lambda<\lambda_{1}$, since the choice of $\Lambda$ is arbitrary.

For each fixed $\lambda \in\left[\Lambda, \lambda_{1}\right.$ [, proposition 5.2 gives the desired existence of a solution, provided $\mathrm{C}_{0}-\mathrm{C}_{3}$ hold. Thereby, we can use a decomposition $H^{1}\left(\mathbb{R}^{N}\right)=Y \oplus Z$, with $Y=\{0\}$.

Concerning $\mathrm{C}_{1}$, we can argue as follows. We make use of the norm $\|u\|_{\lambda}:=$ $\sqrt{B_{\lambda}(u)}$ for all $u \in H^{1}\left(\mathbb{R}^{N}\right)$; recall that this norm is equivalent to the usual one on $H^{1}\left(\mathbb{R}^{N}\right)$. For $\lambda<\lambda_{1}$ kept fixed and for $\|u\|_{\lambda}$ small enough,

$$
\begin{aligned}
I_{\lambda}(u) & \geqslant \frac{1}{2}\|u\|_{\lambda}^{2}-\text { const. }\|u\|_{\lambda}^{2+\sigma} \\
& =\frac{1}{2}\|u\|_{\lambda}^{2} \cdot\left[1-\text { const. }\|u\|_{\lambda}^{\sigma}\right] \\
& \geqslant \frac{1}{4}\|u\|_{\lambda}^{2} .
\end{aligned}
$$

Hence, there exist $\rho_{\lambda}>0$ and $\alpha_{\lambda}>0$ such that

$$
I_{\lambda}(u) \geqslant \alpha_{\lambda}>0 \quad \forall u \in H^{1}\left(\mathbb{R}^{N}\right) \text { with }\|u\|_{H^{1}\left(\mathbb{R}^{N}\right)}=\rho_{\lambda} .
$$

Note that $I_{\lambda}(u)>0$ as long as $0<\|u\|_{H^{1}\left(\mathbb{R}^{N}\right)} \leqslant \rho_{\lambda}$. Thus, $\mathrm{C}_{1}$ holds, with $S_{0}=$ $\left\{u \in H^{1}\left(\mathbb{R}^{N}\right) \mid\|u\|_{H^{1}\left(\mathbb{R}^{N}\right)}=\rho_{\lambda}\right\}$. 
We now examine the condition $\mathrm{C}_{2}$ and we consider the function

$$
s:\left[0,+\infty\left[\rightarrow \mathbb{R}, \quad s(t):=I_{\Lambda}\left(t w_{1}\right)=\frac{1}{2} B_{\Lambda}\left(w_{1}\right) t^{2}-\Phi\left(w_{1}\right) t^{2+\sigma} .\right.\right.
$$

Since both $B_{\Lambda}\left(w_{1}\right)$ and $\Phi\left(w_{1}\right)$ are strictly positive, there must exist some $R>0$, with $I_{\Lambda}\left(R w_{1}\right)<0$. Note that this implies that

$$
I_{\lambda}\left(R w_{1}\right)<0 \quad \forall \lambda \in\left[\Lambda, \lambda_{1}[,\right.
$$

since $I_{\lambda}(u)$ is, as a function of $\lambda$, monotonically non-increasing. We set

$$
\begin{aligned}
\overline{\mathcal{A}}_{0,1} & =\left\{t w_{1} \mid-R \leqslant t \leqslant R\right\} \subset H^{1}\left(\mathbb{R}^{N}\right), \\
\partial \mathcal{A}_{0,1} & =\left\{0, \pm R w_{1}\right\} \\
\mathcal{A}_{0,1} & =\overline{\mathcal{A}}_{0,1} \backslash \partial \mathcal{A}_{0,1} .
\end{aligned}
$$

Then, $\mathrm{C}_{2}$ holds for all $\lambda \in\left[\Lambda, \lambda_{1}\left[\right.\right.$, since $I_{\lambda}(u) \leqslant 0$ for all $u \in \partial \mathcal{A}_{0,1}$.

Note that $\rho_{\lambda}<R$. In this way, we get the oddly linking geometry described in $\S 5$; thereby we consider the linking property with respect to the family $\Gamma_{0,1}$ consisting of all odd homeomorphisms $\gamma: H^{1}\left(\mathbb{R}^{N}\right) \rightarrow H^{1}\left(\mathbb{R}^{N}\right)$, where $\left.\gamma\right|_{\partial \mathcal{A}_{0,1}}$ is the identity mapping. Thus, the context corresponds to the one defined in $\mathrm{C}_{0}$.

Condition $\mathrm{C}_{3}$ holds in view of proposition 4.4.

So we may apply proposition 5.2. For $\lambda \in\left[\Lambda, \lambda_{1}[\right.$ kept fixed,

$$
c_{0,1}(\lambda):=\inf _{\gamma \in \Gamma_{0,1}} \max _{u \in \overline{\mathcal{A}}_{0,1}} I_{\lambda}(\gamma(u))
$$

is a critical value of $I_{\lambda}$ that is bigger than or equal to $\alpha_{\lambda}>0$. And this proves the claim.

\subsection{Existence of multiple critical values}

Before proceeding with the existence of other critical values, we must strengthen assumption $\mathrm{E}_{1}$; for $m=1,2,3, \ldots$, we introduce a new assumption.

Assumption $\mathrm{E}_{m}$. There exists an $m$-dimensional subspace

$$
F_{m}=\operatorname{span}\left\{w_{1}, \ldots, w_{m}\right\} \quad \text { of } H^{1}\left(\mathbb{R}^{N}\right),
$$

with $\Phi(w)>0$ for $w \in F_{m} \backslash\{0\}$. Here, $w_{1}, \ldots, w_{m}$ denotes an orthonormal set in $\left(H^{1}\left(\mathbb{R}^{N}\right),\|\cdot\|_{H^{1}\left(\mathbb{R}^{N}\right)}\right)$.

Proposition 6.2. Suppose that assumptions $L$ and $Q$ are fulfilled. Moreover, suppose that assumption $E_{m}$ holds for some $m \in\{1,2, \ldots\}$.

Then, for any fixed $\Lambda<\lambda_{1}$, there exists a constant $R_{\Lambda}>0$ such that, uniformly for all $\lambda \in\left[\Lambda, \lambda_{1}[\right.$,

$$
I_{\lambda}\left(\sum_{i=1}^{m} \alpha_{i} w_{i}\right)<0 \quad \forall \alpha_{1}, \ldots, \alpha_{1} \in \mathbb{R} \text { with }\left\|\sum_{i=1}^{m} \alpha_{i} w_{i}\right\|_{H^{1}\left(\mathbb{R}^{N}\right)}=R_{\Lambda} .
$$

Proof. Note that

$$
\left\|\sum_{i=1}^{m} \alpha_{i} w_{i}\right\|_{H^{1}\left(\mathbb{R}^{N}\right)}=\sqrt{\sum_{i=1}^{m} \alpha_{i}^{2}} .
$$


We set

$$
A:=\max _{\sum_{i=1}^{m} \alpha_{i}^{2}=1} \frac{B_{\Lambda}}{2}\left(\sum_{i=1}^{m} \alpha_{i} w_{i}\right)>0 \quad \text { and } \quad B:=\min _{\sum_{i=1}^{m} \alpha_{i}^{2}=1} \Phi\left(\sum_{i=1}^{m} \alpha_{i} w_{i}\right)>0 .
$$

Then, for $R>0$,

$$
\max _{\sum_{i=1}^{m} \alpha_{i}^{2}=R^{2}} I_{\Lambda}\left(\sum_{i=1}^{m} \alpha_{i} w_{i}\right) \leqslant A R^{2}-B R^{2+\sigma} .
$$

Thus, we find an $R_{\Lambda}>0$, with

$$
\max _{\sum_{i=1}^{m} \alpha_{i}^{2}=R_{\Lambda}^{2}} I_{\Lambda}\left(\sum_{i=1}^{m} \alpha_{i} w_{i}\right)<0
$$

The claim follows from the remark that, for any $u \in H^{1}\left(\mathbb{R}^{N}\right)$ kept fixed, $I_{\lambda}(u)$ is non-increasing in $\lambda$.

Under the assumptions of the above proposition, we consider

$$
\begin{aligned}
\overline{\mathcal{A}}_{0, m} & :=\left\{\sum_{i=1}^{m} \alpha_{i} w_{i} \mid \alpha_{1}, \ldots, \alpha_{m} \in \mathbb{R}, \sum_{i=1}^{m} \alpha_{i}^{2} \leqslant R_{\Lambda}\right\}, \\
\partial \mathcal{A}_{0, m} & :=\{0\} \cup\left\{\sum_{i=1}^{m} \alpha_{i} w_{i} \mid \alpha_{1}, \ldots, \alpha_{m} \in \mathbb{R}, \sum_{i=1}^{m} \alpha_{i}^{2}=R_{\Lambda}\right\}, \\
\mathcal{A}_{0, m} & :=\overline{\mathcal{A}}_{0, m} \backslash \partial \mathcal{A}_{0, m} .
\end{aligned}
$$

Moreover, we consider the set $\Gamma_{0, m}$ of all odd homeomorphisms $\gamma: H^{1}\left(\mathbb{R}^{N}\right) \rightarrow$ $H^{1}\left(\mathbb{R}^{N}\right)$, where $\left.\gamma\right|_{\partial \mathcal{A}_{0, m}}$ is the identity mapping. For every fixed value $\lambda \in\left[\Lambda, \lambda_{1}[\right.$, we can then apply theorem 5.4 to $I_{\lambda}$. Since the choice of $\Lambda$ is arbitrary, we get the following existence result.

TheOREM 6.3. Suppose that assumptions $L$ and $Q$ hold. Moreover, suppose that assumption $E_{m}$ is fulfilled for some $m \in\{1,2, \ldots\}$.

Then, for each fixed value of $\lambda<\lambda_{1}$, there exist at least $m$ solution pairs $\left(\lambda, \pm u_{j, \lambda}\right)(j=0,1,2, \ldots, m-1)$ of problem $P$.

REMARK 6.4. Let us mention that, for $j=0,1,2, \ldots, m-1$,

$$
0<I_{\lambda}\left( \pm u_{j, \lambda}\right) \leqslant c_{0, m}(\lambda)=\inf _{\gamma \in \Gamma_{0, m}} \max _{\overline{\mathcal{A}}_{0, m}} I_{\lambda}(\gamma(u))
$$

These estimates will be helpful for questions regarding bifurcation.

REMARK 6.5. Suppose that assumptions L and Q hold, and suppose that the function $q$ in assumption $\mathrm{Q}$ is strictly positive. Then, assumption $\mathrm{E}_{m}$ holds for all $m \in \mathbb{N}$ and problem $\mathrm{P}$ has an infinite number of solutions for each $\lambda<\lambda_{1}$.

\subsection{Bifurcation of solutions from $\lambda_{1}$}

In order to discuss bifurcation, we introduce an assumption that reinforces assumption $\mathrm{E}_{1}$. 
Assumption $E_{1}^{\text {bif }}$. Assumption $\mathrm{E}_{1}$ holds with $F_{1}=\operatorname{span}\left\{w_{1}\right\}$, where $w_{1}$ is an eigenfunction of $-\Delta+V$ corresponding to the eigenvalue $\lambda_{1}$.

For $\lambda \in\left[\lambda_{1}-1, \lambda_{1}\left[\right.\right.$, we consider the aforementioned critical value $c_{0,1}(\lambda)$, constructed with the help of the space $F_{1}$, given in assumption $\mathrm{E}_{1}^{\text {bif }}$.

Proposition 6.6. Suppose that assumptions $L, Q$ and $E_{1}^{\text {bif }}$ hold.

Then, we get the following.

(1) The critical level $c_{0,1}(\lambda)$ depends in a non-increasing way on $\lambda \in\left[\lambda_{1}-1, \lambda_{1}[\right.$.

(2) Moreover, we have that

$$
0<c_{0,1}(\lambda) \leqslant \text { const. }\left(\lambda_{1}-\lambda\right)^{1+2 / \sigma},
$$

so $\lim _{\lambda \rightarrow \lambda_{1}^{-}} c_{0,1}(\lambda)=0$.

(3) For $\lambda^{(1)}$ and $\lambda^{(1)} \in\left[\lambda_{1}-1, \lambda_{1}\left[\right.\right.$, with $\lambda^{(1)}<\lambda^{(2)}$, we have that

$$
I_{\lambda(1)}(v)-I_{\lambda(2)}(v)=\frac{1}{2}\left(\lambda^{(2)}-\lambda^{(1)}\right)|v|_{L^{2}\left(\mathbb{R}^{N}\right)} \quad \forall v \in H^{1}\left(\mathbb{R}^{N}\right) .
$$

Proof. The first point follows from the observation that, for $u$ kept fixed, $I_{\lambda}(u)$ is non-increasing as a function depending on $\lambda$.

Concerning the second point, we first use the property that $w_{1}$ is an eigenfunction, so

$$
B_{\lambda}\left(w_{1}\right)=\underbrace{B_{\lambda_{1}}\left(w_{1}\right)}_{=0}+\left|w_{1}\right|_{L^{2}\left(\mathbb{R}^{N}\right)}^{2}\left(\lambda_{1}-\lambda\right) .
$$

The function $s(t):=I_{\lambda}\left(t w_{1}\right)$ admits its maximal value for

$$
t=t_{1}:=\left[\frac{\left|w_{1}\right|_{L^{2}\left(\mathbb{R}^{N}\right)}^{2}}{(2+\sigma) \Phi\left(w_{1}\right)}\right]^{1 / \sigma}\left(\lambda_{1}-\lambda\right)^{1 / \sigma}
$$

an easy computation shows that $s\left(t_{1}\right)=$ const. $\left(\lambda_{1}-\lambda\right)^{1+2 / \sigma}$. Hence,

$$
0<c_{0,1}(\lambda) \leqslant \max _{u \in \overline{\mathcal{A}}_{1}} I_{\lambda}(u)=s\left(t_{1}\right)=\text { const. } \cdot\left(\lambda_{1}-\lambda\right)^{1+2 / \sigma} .
$$

The last point follows from

$$
I_{\lambda^{(1)}}(v)-I_{\lambda^{(1)}}(v)=B_{\lambda^{(1)}}(v)-B_{\lambda^{(2)}}(v)=\left(\lambda^{(2)}-\lambda^{(1)}\right)|v|_{L^{2}\left(\mathbb{R}^{N}\right)} .
$$

We now apply the abstract results derived in $\S 5$, and, more specifically, theorem 5.7, with $n=0, \lambda^{-}=\lambda_{1}-1$ and $\lambda^{+}=\lambda_{1}$.

Theorem 6.7. Suppose that assumptions $L, Q$ and $E_{1}^{\text {bif }}$ hold. Then, $\lambda_{1}$ is an $L^{2}-$ bifurcation point of problem $P$.

REMARK 6.8. If the function $q$ in assumption $\mathrm{Q}$ is strictly positive on an at least sufficiently large ball of centre 0 in $\mathbb{R}^{N}$, then assumption $E_{1}^{\text {bif }}$ holds and we have bifurcation. 


\section{Existence of multiple solutions in spectral gaps}

In this section we analyse the existence of solutions for problem $\mathrm{P}$ when $\lambda \in$ ]$\lambda_{1}, 0[\backslash \sigma(-\Delta+V)$. For such a $\lambda$, there exists some $i=i(\lambda)$, with

$$
\left.\left.\lambda_{i}<\lambda \text { and }\right] \lambda_{i}, \lambda\right] \cap \sigma(-\Delta+V)=\emptyset
$$

We set $\lambda^{-}=\lambda_{i}$ and we consider the spectral gap $] \lambda^{-}, \lambda^{+}[$containing $\lambda$. Note that $\lambda^{+}=\lambda_{i+1}$ except if, in assumption $\mathrm{L}$, we have that $L \neq \mathbb{N}$ and $i=\ell$; in this latter case we have that $\lambda^{+}=0$.

Inside the spectral gap $] \lambda^{-}, \lambda^{+}$, we can replace the usual norm $\|\cdot\|_{H^{1}\left(\mathbb{R}^{N}\right)}$ by the equivalent norm $\|\cdot \cdot\|_{\lambda}$ defined in $\S 4$. Recall that the corresponding scalar product is

$$
((u, v))_{\lambda}=\left(\left(L_{1}+V-\lambda \mathbb{I}\right)(Q-P) u, v\right)_{L^{2}\left(\mathbb{R}^{N}\right)},
$$

where the projections $P$ and $Q$ are defined in $\S 4$.

Proposition 7.1. Suppose that assumption L holds and that $\lambda$ is inside the spectral gap $] \lambda^{-}, \lambda^{+}\left[\right.$. Let $u_{n}$ be an eigenfunction corresponding to an eigenvalue $\lambda_{n}$ of $-\Delta+$ $V$, and let $u_{m}$ be an eigenfunction corresponding to another eigenvalue $\lambda_{m}$ of $-\Delta+$ $V$.

The eigenfunctions $u_{n}$ and $u_{m}$ are then orthogonal with respect to the $L^{2}$-scalar product as well as with respect to the scalar product $((\cdot, \cdot))_{\lambda}$.

Proof. Multiplying $-\Delta u_{n}+V u_{n}=\lambda_{n} u_{n}$ by $u_{m}$ and $-\Delta u_{m}+V u_{m}=\lambda_{m} u_{m}$ by $u_{n}$ gives

$$
0=\left(\lambda_{n}-\lambda_{m}\right) \int_{\mathbb{R}^{N}} u_{n} u_{m} \mathrm{~d} x ;
$$

the orthogonality in $L^{2}$ follows, since $\lambda_{n} \neq \lambda_{m}$. Moreover,

$$
\int_{\mathbb{R}^{N}}\left(\nabla u_{n} \cdot \nabla u_{m}+V u_{n} u_{m}\right) \mathrm{d} x=0
$$

proves the claim, since $(Q-P) u_{m}= \pm u_{m}$.

\subsection{A positivity result}

When we have defined critical values as in $\S 5$, we can observe that the behaviour of $I_{\lambda}$ in a vicinity of 0 plays a major role. When we are in a spectral gap $] \lambda^{-}, \lambda^{+}[$, we need a positivity result of the following kind.

Proposition 7.2. Suppose that assumptions $L$ and $Q$ hold and that $\lambda \in] \lambda^{-}, \lambda^{+}[$.

Then, there exists $\rho_{\lambda}>0, \alpha_{\lambda}>0$ such that $I_{\lambda}(u) \geqslant \alpha_{\lambda}$ for all $u \in Q\left(H^{1}\left(\mathbb{R}^{N}\right)\right)$, with $\|u\|_{H^{1}\left(\mathbb{R}^{N}\right)}=\rho_{\lambda}$. Moreover, $I_{\lambda}(u)>0$ for $u \in Q\left(H^{1}\left(\mathbb{R}^{N}\right)\right)$, with

$$
0<\|u\|_{H^{1}\left(\mathbb{R}^{N}\right)} \leqslant \rho_{\lambda} .
$$

Proof. For $u \in Q\left(H^{1}\left(\mathbb{R}^{N}\right)\right)$, we have that

$$
B_{\lambda}(u)=\|u\|_{\lambda}^{2}=\left(\left(L_{1}+V-\lambda \mathbb{I}\right) u, u\right)_{L^{2}\left(\mathbb{R}^{N}\right)} .
$$


By the equivalence of norms, we get that

$$
\begin{aligned}
I_{\lambda}(u) & \geqslant \text { const. }\|u\|_{\lambda}^{2}-\text { const. }\|u\| \|_{\lambda}^{2+\sigma} \\
& \geqslant \text { const. }\|u\|_{H^{1}\left(\mathbb{R}^{N}\right)}^{2+}-\text { const. }\|u\|_{H^{1}\left(\mathbb{R}^{N}\right)}^{2+\sigma} \\
& =\text { const. }\|u\|_{H^{1}\left(\mathbb{R}^{N}\right)}^{2}\left[1-\text { const. }\|u\|_{H^{1}\left(\mathbb{R}^{N}\right)}^{\sigma}\right] .
\end{aligned}
$$

This proves the claim.

We can use the abstract setting of $\S 5$ if we set

$$
X:=H^{1}\left(\mathbb{R}^{N}\right), \quad Y:=P\left(H^{1}\left(\mathbb{R}^{N}\right)\right) \quad \text { and } \quad Z:=Q\left(H^{1}\left(\mathbb{R}^{N}\right)\right) .
$$

Moreover, we need the 'circle' $S_{L^{-}}:=\left\{z \in Z \mid\|z\|_{H^{1}\left(\mathbb{R}^{N}\right)}=\rho_{\lambda}\right\}$, where $\rho_{\lambda}$ is given by the above proposition.

Before proceeding further, we introduce some notation, supposing that assumption L holds. For each eigenvalue $\lambda_{j} \in \sigma(-\Delta+V)$, we denote by $E\left(\lambda_{j}\right)$ the corresponding eigenspace, and we set $l_{j}:=\operatorname{dim} E\left(\lambda_{j}\right)$. Moreover, we set $L_{j}:=l_{1}+\cdots+l_{j}$. When $\lambda \in] \lambda^{-}, \lambda^{+}\left[\right.$, we set $L^{-}:=L_{i(\lambda)}$, where $i(\lambda)$ is such that $\lambda^{-}=\lambda_{i(\lambda)}$.

\subsection{Existence of multiple solutions in a gap}

Our aim is to establish the existence of an infinite number of critical values of $I_{\lambda}$ for $\lambda \in]-\infty, \lambda^{+}\left[\backslash \sigma(-\Delta+V)\right.$. To achieve this, we choose some $\Lambda$, with $\Lambda<\lambda^{-}$, and we define, for each fixed value of $\lambda \in\left[\Lambda, \lambda^{+}[\backslash \sigma(-\Delta+V)\right.$, a countable number of critical values. Since the choice of $\Lambda$ is arbitrary, we can reach the desired conclusion.

As a first step, we will show that if assumption $\mathrm{E}_{k}$ holds for some $k \in\left\{L^{-}+1\right.$, $\left.L^{-}+2, \ldots\right\}$ and if $Y \subset F_{k}$, problem $\mathrm{P}$ has (at least) $k-L^{-}$solution pairs $(\lambda, \pm u)$ for the corresponding value of $\lambda$. This in turn will imply the existence of an infinite number of solution pairs, if assumption $\mathrm{E}_{k}$ holds for all values of $k>L^{-}$.

An analysis of dimensions shows that $\operatorname{dim}\left(F_{k} \cap Z\right)=k-L^{-}$. We can apply the results of $\S 5$ if we set

$$
n=L^{-} \quad \text { and } \quad m:=k-L^{-},
$$

and if we choose orthonormal elements $w_{1}, \ldots, w_{m}$ in $F_{k} \cap Z$. Indeed, we may define the sets

$$
\begin{aligned}
\overline{\mathcal{A}}_{n, m} & :=\left\{x=y+\sum_{j=1}^{m} \alpha_{j} w_{j} \mid y \in Y,\|x\|_{H^{1}\left(\mathbb{R}^{N}\right)} \leqslant R_{\Lambda}\right\}, \\
\partial \mathcal{A}_{n, m} & :=\{0\} \cup\left\{x=y+\sum_{j=1}^{m} \alpha_{j} w_{j} \mid y \in Y,\|x\|_{H^{1}\left(\mathbb{R}^{N}\right)}=R_{\Lambda}\right\}, \\
\mathcal{A}_{n, m} & :=\overline{\mathcal{A}}_{n, m} \backslash \partial \mathcal{A}_{n, m},
\end{aligned}
$$

where we choose $R_{\Lambda}$ in order to have $\left.I_{\lambda}\right|_{\partial \mathcal{A}_{n, m}} \leqslant 0$. According to the following proposition, such a choice for $R_{\Lambda}$ is possible.

Proposition 7.3. Suppose that assumptions $L$ and $Q$ hold. Furthermore, suppose that assumption $E_{k}$ holds for some $k>L^{-}$and that $Y \subset F_{k}$.

Then, there exists, for any fixed value of $\Lambda<\lambda^{-}$, a constant $R_{\Lambda}>0$ such that

$$
\left.I_{\lambda}\right|_{\partial \mathcal{A}_{n, m}} \leqslant 0 \quad \forall \lambda \in\left[\Lambda, \lambda^{+}[.\right.
$$


Proof. We put $K:=\operatorname{span}\left\{Y, w_{1}, \ldots, w_{m}\right\}$ and we define

$$
\begin{aligned}
& A:=\max \left\{\frac{1}{2} B_{\Lambda}(u) \mid u \in K,\|u\|_{H^{1}\left(\mathbb{R}^{N}\right)}=1\right\} \\
& B:=\min \left\{\Phi(u) \mid u \in K,\|u\|_{H^{1}\left(\mathbb{R}^{N}\right)}=1\right\} .
\end{aligned}
$$

Then, we conclude as in the proof of proposition 6.2 .

We now set, for $j=0, \ldots, m-1$,

$$
d_{n, m, j}(\lambda):=\inf _{\gamma \in \Gamma_{n, m}} \inf _{U \in G_{j}} \max _{\mathcal{A}_{n, m} \backslash U} I(\gamma(u)) .
$$

The set $\Gamma_{n, m}$ contains all the odd homeomorphisms $\gamma: H^{1}\left(\mathbb{R}^{N}\right) \rightarrow H^{1}\left(\mathbb{R}^{N}\right)$, where $\left.\gamma\right|_{\partial \mathcal{A}_{n, m}}$ is the identity. Moreover, we set $G_{0}=\{\emptyset\}$ and

$$
G_{j}:=\left\{U \subset H^{1}\left(\mathbb{R}^{N}\right) ; \mid U=-U, 0 \notin \bar{U} \text {, genus of } U \leqslant j\right\} .
$$

We then obtain the following result (see proposition 5.4).

THEOREM 7.4. Suppose that assumptions L and $Q$ hold. Furthermore, suppose that assumption $E_{k}$ holds for some $k>L^{-}$and that $Y \subset F_{k}$.

Then, there exist, for all $\lambda \in]-\infty, \lambda^{+}\left[\backslash \sigma(-\Delta+V)\right.$, at least $k-L^{-}$solution pairs $\left(\lambda, u_{i ; \lambda}\right)$ of problem P. For $i=L^{-}+1, L^{-}+2, \ldots, k$, we have that

$$
I_{\lambda}^{\prime}\left(u_{i ; \lambda}\right)=0, \quad 0<I_{\lambda}\left(u_{i ; \lambda}\right)=d_{n, m, i}(\lambda)
$$

Corollary 7.5. Suppose that assumptions $L$ and $Q$ hold. Moreover, suppose that assumption $E_{k}$ holds, for all $k>L^{-}$, and that $Y \subset F_{k}$. (This is the case if, for example, $q$ is strictly positive everywhere.)

Then, there exist, for all $\lambda \in]-\infty, \lambda^{+}[\backslash \sigma(-\Delta+V)$, an infinite number of solution pairs $\left(\lambda, u_{i ; \lambda}\right)$ of problem P. For $i=L^{-}+1, L^{-}+2, \ldots$, we have that

$$
I_{\lambda}^{\prime}\left(u_{i ; \lambda}\right)=0, \quad 0<I_{\lambda}\left(u_{i ; \lambda}\right)=d_{n, m, i}(\lambda)
$$

\subsection{Bifurcation from eigenvalues}

We now discuss the bifurcation from the eigenvalue $\lambda^{+}=\lambda_{i+1}$, with $i \geqslant 1$. Solutions will exist for $\lambda<\lambda_{i+1}$ if we assume $\mathrm{E}_{m}$ for $m=L^{-}+1$. We will discuss bifurcation under the following, stronger assumption.

Assumption $\mathrm{E}_{L^{-}+1}^{\mathrm{bif}}$. This assumption holds with

$$
F_{L^{-}+1}=\operatorname{span}\left\{w_{1}, w_{2}, \ldots, w_{L^{-}}, w_{L^{-}+1}\right\},
$$

where $w_{1}, \ldots, w_{L^{-}}$are eigenfunctions of $-\Delta+V$ corresponding to the eigenvalues $\lambda_{1}<\cdots<\lambda_{i}$, and $w_{L^{-+1}}$ is an eigenfunction of $-\Delta+V$ corresponding to the eigenvalues $\lambda_{i+1}=\lambda^{+}$.

This assumption holds if $q$ is strictly positive on a sufficiently large ball of centre 0 in $\mathbb{R}^{N}$.

We intend to apply the abstract result of theorem 5.7. To accomplish this, we need to verify that assumptions $\mathrm{B}_{1}-\mathrm{B}_{3}$ hold. 
Proposition 7.6. Suppose that assumptions $L$ and $Q$ hold, as well as assumption $E_{L^{-}+1}^{\text {bif }}$. We consider the critical value $c_{n, 1}(\lambda)$, for $\left.\lambda \in\right] \lambda^{-}, \lambda^{+}[$.

Then, we have the following.

(1) $c_{n, 1}(\lambda)$ is non-increasing, while $\lambda$ varies inside $] \lambda^{-}, \lambda^{+}[$.

(2) For $\lambda \in] \lambda^{-}, \lambda^{+}[$, we have that

$$
0<c_{n, 1}(\lambda) \leqslant \text { const. }\left(\lambda^{+}-\lambda\right)^{1+2 / \sigma},
$$

so

$$
\lim _{\lambda \rightarrow \lambda^{+}, \lambda<\lambda^{+}} c_{n, 1}(\lambda)=0 .
$$

Moreover, the derivative $c_{n, 1}^{\prime}(\lambda)$ exists a.e. on $] \lambda^{-}, \lambda^{+}[$and, whenever this derivative exists, we have that $c_{n, 1}^{\prime}(\lambda) \leqslant 0$.

(3) For $\lambda^{(1)}$ and $\left.\lambda^{(1)} \in\right] \lambda^{-}, \lambda^{+}\left[\right.$, with $\lambda^{(1)}<\lambda^{(2)}$, we have that

$$
I_{\lambda^{(1)}}(v)-I_{\lambda^{(2)}}(v)=\frac{1}{2}\left(\lambda^{(2)}-\lambda^{(1)}\right)|v|_{L^{2}\left(\mathbb{R}^{N}\right)} \quad \forall v \in H^{1}\left(\mathbb{R}^{N}\right) .
$$

Proof. The first part follows from the fact that $I_{\lambda}$ is non-increasing in $\lambda$.

To prove the second statement, we show that

$$
\max _{w \in F_{L-+1}} I_{\lambda}(w) \leqslant \operatorname{const} .\left(\lambda^{+}-\lambda\right)^{1+2 / \sigma} .
$$

Note that, for any $w \in F_{L^{-}+1}$, we have that

$$
B_{\lambda}(w)=B_{\lambda^{+}}(w)+\left(\lambda^{+}-\lambda\right)|w|_{L^{2}\left(\mathbb{R}^{N}\right)}^{2} .
$$

We can now estimate $\max _{w \in F_{L-+1}} I_{\lambda}(w)$ in the following way. One can find an element $\bar{w} \in F_{L^{-}+1}$, with $|\bar{w}|_{L^{2}\left(\mathbb{R}^{N}\right)}=1$ and

$$
\max _{w \in F_{L^{-}+1}} I_{\lambda}(w)=\max _{t \geqslant 0} I_{\lambda}(t \bar{w}) .
$$

Since $\inf \left\{\left.\Phi(w)\left|w \in F_{L^{-}+1},\right| w\right|_{L^{2}\left(\mathbb{R}^{N}\right)}=1\right\}=: m>0$, one gets that

$$
\max _{t \geqslant 0} I_{\lambda}(t \bar{w}) \leqslant \max _{t \geqslant 0} \frac{1}{2}\left(\lambda^{+}-\lambda\right) t^{2}-m t^{2+\sigma} .
$$

This latter maximum can be computed, since it is achieved when

$$
t=\left(\frac{\lambda^{+}-\lambda}{(2+\sigma) m}\right)^{1 / \sigma}
$$

One thus gets the desired claim that

$$
\max _{w \in F_{L^{-}+1}} I_{\lambda}(w) \leqslant \operatorname{const.}\left(\lambda^{+}-\lambda\right)^{1+2 / \sigma} .
$$

The last part follows as in proposition 6.6.

Applying theorem 5.7, one gets the following bifurcation result. 
THEOREM 7.7. Suppose that assumptions $L$ and $Q$ hold, as well as assumption $E_{L^{-}+1}^{\mathrm{bif}}$, with $i \geqslant 1$.

Then, $\lambda^{+}=\lambda_{i+1}$ is an $L^{2}$-bifurcation point for problem $P$.

We can even address the problem of multiple bifurcation by applying theorem 5.8. To this end, we introduce the following assumption, where $m$ belongs to $\left\{1,2, \ldots, l_{i+1}\right\}$.

Assumption $\mathrm{E}_{L^{-}+m}^{\mathrm{bif}}$. This assumption holds with the special choice of

$$
F_{L^{-}+m}=\operatorname{span}\left\{w_{1}, w_{2}, \ldots, w_{L^{-}}, \ldots, w_{L^{-}+1}, \ldots, w_{L^{-}+m}\right\},
$$

where $w_{1}, \ldots, w_{L^{-}}$are eigenfunctions of $-\Delta+V$ corresponding to the eigenvalues $\lambda_{1}<\cdots<\lambda_{i}$ and $w_{L^{-+1}}, \ldots, w_{L^{-+}}$are eigenfunctions of $-\Delta+V$ corresponding to the eigenvalues $\lambda_{i+1}=\lambda^{+}$.

Note that this assumption holds if, for example, $q$ is strictly positive.

TheOREM 7.8. Suppose that assumptions $L$ and $Q$ hold, as well as assumption $E_{L^{-}+m}^{\mathrm{bif}}$, for some $m \in\left\{1,2, \ldots, l_{i+1}\right\}(i \geqslant 1)$.

Then, $\lambda^{+}=\lambda_{i+1}$ is an $L^{2}$-bifurcation point of multiplicity $m$ (at least) for problem $P$.

Let us end with a remark. If in assumption $\mathrm{L}$ we have $L=\mathbb{N}$ and if the above theorem remains valid for each gap $] \lambda_{i}, \lambda_{i+1}[$, then the infimum of the essential spectrum is also a bifurcation point.

REMARK 7.9. As already mentioned, the variational characterization of critical levels $c_{n, m}$ and $d_{n, m, k}$ can be extended to situations where $\operatorname{dim} Y=\infty$. This will be done in [12], by considering a Schrödinger equation with periodic functions $V$ and $q$. This kind of potential makes everything more difficult. The usual deformation theorems can no longer be applied and the Palais-Smale condition no longer holds.

\section{Acknowledgements}

We express our gratitude to the referee for helpful remarks concerning the presentation of the results.

\section{References}

1 A. Ambrosetti and P. H. Rabinowitz. Dual variational methods in critical point theory and applications. J. Funct. Analysis 14 (1973), 259-381.

2 S.-N. Chow and J. K. Hale. Methods of bifurcation theory, Grundlehren der Mathematischen Wissenschaften, vol. 251 (Springer, 1982).

3 R. Dautray and J.-L. Lions. Mathematical analysis and numerical methods for science and technology: spectral theory and applications (Springer, 1990).

4 J. Giacomoni and L. Jeanjean. A variational approach to bifurcation into spectral gaps. Annali Scuola Norm. Sup. Pisa IV 28 (1999), 651-674.

5 H.-P. Heinz. Lacunary bifurcation for operator equations and nonlinear boundary value problems on $\mathbf{R}^{N}$. Proc. R. Soc. Edinb. A 118 (1991), 237-270.

6 Y. Jabri. The mountain pass theorem: variants, generalizations and some applications, Encyclopedia of Mathematics and Its Applications, vol. 95 (Cambridge University Press, 2003). 
$7 \quad$ R. Jost. Quantenmechanik 1 (Verlag der Fachvereine an der ETH-Z, 1973).

8 T. Kato. Perturbation theory for linear operators, Classics in Mathematics (Springer, 1995).

9 W. Kryszewski and A. Szulkin. Generalized linking theorem with an application to a semilinear Schrödinger equation. Adv. Diff. Eqns 3 (1998), 441-472.

10 H.-J. Ruppen. Mulitplicity results for a semilinear, elliptic differential equation with conflicting nonlinearities. J. Diff. Eqns 147 (1998), 79-122.

11 H.-J. Ruppen. Conflicting nonlinearities and spectral lacunae bounded on one side by eigenvalues. Proc. R. Soc. Edinb. A 135 (2005), 1041-1072.

12 H.-J. Ruppen. A generalized min-max theorem for functionals of strongly indefinite sign. Calc. Var. PDEs (In the press.)

13 H.-J. Ruppen. A generalized mountain-pass theorem for functionals of strongly indefinite sign. (In preparation.)

14 M. Struwe. Variational methods: applications to nonlinear partial differential equations and Hamiltonian systems (Springer, 1990).

15 C. A. Stuart. Bifurcation for Neumann problems without eigenvalues. J. Diff. Eqns 36 (1980), 391-407.

16 C. A. Stuart. Bifurcation from the continuous spectrum in the $L^{2}$-theory of elliptic equations on $\mathbf{R}^{n}$. Recent methods in nonlinear analysis and applications, Proc. SAFA Conference IV, pp. 231-300 (Naples, Liguori, 1981).

17 C. A. Stuart. Bifurcation for Dirichlet problems without eigenvalues. Proc. Lond. Math. Soc. 45 (1982), 169-192.

18 C. A. Stuart. Bifurcation in $L^{p}\left(\mathbb{R}^{N}\right)$ for a semilinear elliptic equation. Proc. Lond. Math. Soc. 57 (1988), 511-541.

19 C. A. Stuart. Bifurcation into spectral gaps. Bull. Belg. Math. Soc. Simon Stevin 2 (suppl.) (1995), 59 .

(Issued 4 October 2013) 